\title{
Zonal Harmonic Perturbations of an Accurate Reference Orbit of an Artificial Satellite*
}

\author{
John P. Vinti \\ (July 24, 1963)
}

\begin{abstract}
The theory developed in an earlier paper, for an accurate reference orbit of an artificial satellite, is first slightly modified, so as to prepare the way for a treatment of zonal harmonic perturbations. Delaunay variables are next introduced, by means of certain linear combinations of the action variables, along with their canonical conjugates. Application of the von Zeipel method then permits the calculation of the most important zonal harmonic perturbations. These arise from the third, with coefficient $J_{3}$, and the residual fourth, with coefficient $J_{4}+J_{2}^{2}$. The accuracy of the secular and shortperiodic effects is through terms of order $J_{2}^{2}$ and that of the long-periodic effects is through terms of order $J_{2}$. Since the reference orbit itself, with its exact secular terms, takes care of all but 0.5 percent of the deviation of the earth's gravitational field from spherical symmetry, the overall secular accuracy of the final orbit surpasses that of other second order theories. The results are compared with those of Kozai.
\end{abstract}

\section{Reference Orbit}

The author [Vinti 1959a,b] has introduced a potential

$$
V^{\prime}=-\mu \rho\left(\rho^{2}+c^{2} \eta^{2}\right)^{-1}
$$

that can represent accurately the gravitational field of an oblate planet. Here $\mu$ is the product of the gravitational constant $G$ and the planet's mass, $c$ is an adjustable length, and $\rho$ and $\eta$ are oblate spheroidal coordinates, defined by the equations

$$
\begin{gathered}
X+i Y=r \cos \theta \exp i \phi=\left[\left(\rho^{2}+c^{2}\right)\left(1-\eta^{2}\right)\right]^{1 / 2} \exp i \phi \\
Z=r \sin \theta=\rho \eta .
\end{gathered}
$$

If an artificial satellite is at the field point, $r, \theta$, and $\phi$ are respectively its planetocentric distance, declination, and right ascension, and $X, Y$, and $Z$ are its rectangular coordinates, $O Z$ being along the planet's axis and $O X$ pointing toward its vernal equinox.

If $r_{e}$ is the equatorial radius, the true potential is

$$
V=-\mu r^{-1}\left[1-\sum_{n=2}^{\infty}\left(r_{e} / r\right)^{n} J_{n} P_{n}(\sin \theta)\right]+\text { tesseral harmonics, }
$$

where only the products $r_{e}^{n} J_{n}$ need to be known. That is, differences in the definition of $r_{e}$, when noncircularity of the equator is taken into account, can be reconciled by small adjustments of the $J$ 's. Then if

$$
c=r_{e} J_{2}^{1 / 2},
$$

${ }^{*}$ Research supported by the National Aeronautics and Space Agency, U.S.A. 
$V^{\prime}$ represents exactly the zeroth harmonic $-\mu / r$ and the second harmonic and also gives higher even harmonics, characterized by

$$
J_{2 m}=(-1)^{m+1} J_{2}^{m}
$$

In particular it gives $J_{4}+J_{2}^{2}=0$, as compared with observed values for the earth ranging from $-(0.9) 10^{-6}$ to $(0.4) 10^{-6}$ (Kaula 1962; King-Hele, Cook, and Rees 1963). Consequently it accounts for about 99.5 percent of the deviation of $V$ from the value $-\mu / r$ corresponding to spherical symmetry. It thus accounts almost completely for the flattening of the earth, leading to a geoid that never departs by more than about $30 \mathrm{~m}$ from the true sea-level surface.

For the drag-free motion of an artificial satellite the potential (1.00) leads to a separable problem, which has been worked out analytically [Vinti 1961 a,b, 1962]. This solution, holding for all angles of inclination and containing no critical inclination or long-periodic terms, gives secular terms exactly by means of rapidly converging infinite series and short-periodic terms correctly through order $J_{2}^{2}$. We call this orbit corresponding to (1.00) the reference orbit. For such a reference orbit error can never accumulate, because of the exactness of the secular terms, and the periodic terms can be in error only by amounts of the order $J_{2}^{3}$, i.e., by about 1 part in $10^{9}$, since $J_{2}=(1.08) 10^{-3}$ for the earth.

\section{Zonal Harmonic Perturbations}

For a satellite of the earth, if its orbit is high enough so that drag is small and low enough so that the moon's effect is small, the above reference orbit ought to hold rather well for a good many revolutions. (I purposely choose vague words here, since numerical comparisons are still incomplete.) Eventually, however, the actual orbit will deviate more and more from such a reference orbit, because of the neglected forces. These include forces arising from drag, meteoritic impact, radiation, electromagnetic fields, the sun, and the moon, and the neglected part of the earth's gravitational potential, corresponding to (1.03) minus (1.00). Since the expansion of (1.00) in zonal harmonics is

$$
V^{\prime}=-\mu r^{-1} \sum_{m=1}^{\infty}\left(r_{e} / r\right)^{2 m}\left(-J_{2}\right)^{m} P_{2 m}(\sin \theta)
$$

this difference is

$$
\begin{aligned}
V-V^{\prime}=\mu r^{-1}\left[\left(\frac{r_{e}}{r}\right)^{3} J_{3} P_{3}(\sin \theta)+\left(\frac{r_{e}}{r}\right)^{4}\right. & \left(J_{4}+J_{2}^{2}\right) P_{4}(\sin \theta)+\left(\frac{r_{e}}{r}\right)^{5} J_{5} P_{5}(\sin \theta) \\
& \left.+\left(\frac{r_{e}}{r}\right)^{6}\left(J_{6}-J_{2}^{3}\right) P_{6}(\sin \theta)+\ldots\right]+ \text { tesseral harmonics. }
\end{aligned}
$$

Of these forces the most important, for any satellite with a large ratio of mass to area, are the forces corresponding to $J_{3}$ and $J_{4}+J_{2}^{2}$ in $(2.01)$ and drag, which as determined empirically may include effects of meteoritic impact. For a double satellite [Langer and Vinti 1963] only (2.01) and the lunar-solar perturbation remain.

The purpose of the present paper is to devise a method for correcting for the effects of any of the zonal harmonics in (2.01). The first example considered is the residual fourth harmonic, with coefficient $J_{4}+J_{2}^{2}$. This harmonic leads not only to short-periodic effects and secular effects, but also to long-periodic effects depending on a resonance denominator $1-5 \cos ^{2} I$, giving rise to a critical inclination $I=63.4^{\circ}$. The second example considered is the third harmonic, with coefficient $J_{3}$. This gives rise only to short-periodic effects and to long-periodic effects without singularities, so that it is qualitatively less interesting. Because of its greater magnitude, however, $J_{3}$ being about $(-2.4) 10^{-6}$ and $\left|J_{4}+J_{2}^{2}\right|$ being probably somewhat less than $(0.5) 10^{-6}$ [Kaula 1962; King-Hele, Cook, and Rees 1963], it leads to somewhat larger periodic effects. 


\section{The Dynamical Problem}

Our problem is thus to find the motion of a satellite, taken to be of unit mass, when the Hamiltonian is

$$
F=-T+\mu \rho\left(\rho^{2}+c^{2} \eta^{2}\right)^{-1}+F_{1},
$$

where $T$ is its kinetic energy and where, for the residual fourth harmonic

$$
\begin{aligned}
& F_{1}=-\mu r_{e}^{4} r^{-5} \sigma_{4} P_{4}(\sin \theta) \\
& \sigma_{4} \equiv J_{4}+J_{2}^{2}
\end{aligned}
$$

(We have here reversed the sign of the Hamiltonian, to agree with the usual practice with Delaunay variables.) In carrying out this solution we shall use the results and notation of the solution [Vinti $1961 \mathrm{a}, \mathrm{b}, 1962]$ for the reference orbit, for which $F^{\prime}=0$. If in (3.02) we then put

$$
\begin{gathered}
r=a(1-e \cos E)=a\left(1-e^{2}\right)(1+e \cos v)^{-1} \\
\sin \theta=\sin I \sin \left(v+\beta_{2}\right),
\end{gathered}
$$

the expression (3.02) for $F_{1}$ will be correct through order $J_{2}^{2}$. To this order of accuracy the anomalies $E$ and $v$ may be given by the quasi-elliptic expressions

$$
E \approx M_{s}+E_{0} \quad(3.06) \quad v \approx M_{s}+v_{0} .
$$

Note that (3.04) corresponds to $r \approx \rho$ and (3.05) to other approximations of zeroth order in $J_{2}$, viz,

$$
\psi \approx \psi_{s}+\psi_{0} \approx M_{s}+\beta_{2}+v_{0} \approx v+\beta_{2} \quad(3.08) \quad \eta \approx \sin \theta
$$

Such an order of accuracy will result in errors of order $J_{2}^{3}$ for those secular and short-periodic effects which are produced by the perturbing potential (3.02) and of order $J_{2}^{2}$ for the corresponding long-periodic effects. This perturbation (3.02) represents about 0.1 percent of the departure of the earth from sphericity. The solution for the other harmonics in (2.01) will have the same accuracy. However, since all of these higher harmonics represent only about 0.5 percent of the earth's departure from sphericity, their lower accuracy, as compared with that of the reference orbit which has already accounted for 99.5 percent of this departure, should not result in serious cumulative errors.

In doing the perturbation theory, the first canonical variables that come to mind are the Jacobi "constants," viz, the $\alpha$ 's and $\beta$ 's of the reference orbit. When the reference orbit is elliptic, however, their shortcomings are well known and they lead to the same troubles in the present problem, giving rise to Poisson terms, linear in the time, in the variations in $\alpha_{1}$ and $\alpha_{2}$.

The next set of canonical variables that one might try is the set generated from the $\alpha$ 's and $\beta$ 's by the generating function

$$
S^{\prime}=-\alpha_{1} t+\mu\left(-2 \alpha_{1}\right)^{-1 / 2} \beta_{1}^{\prime}+\alpha_{2} \beta_{2}^{\prime}+\alpha_{3} \beta_{3}^{\prime} .
$$

If we define $n_{0}$ by

$$
\mu=n_{0}^{2} a_{0}^{3} \quad a_{0} \equiv-\frac{1}{2} \mu / \alpha_{1},
$$


the resulting canonical variables are

$$
\begin{array}{cc}
L=\left(\mu a_{0}\right)^{1 / 2} & l=n_{0}\left(t+\beta_{1}\right) \\
\alpha_{2} & \beta_{2} \\
\alpha_{3} & \beta_{3},
\end{array}
$$

canonical with respect to the Hamiltonian

$$
F=\frac{1}{2} \cdot \mu^{2} / L^{2}+F_{1}
$$

When the reference orbit is elliptic, this set is the same as the fast Delaunay set [Garfinkel 1960].

One may then attempt to apply the von Zeipel method in the way successfully used by Brouwer [1959] and Garfinkel [1959], first eliminating short-periodic terms and then proceeding to eliminate long-periodic terms. One finds, however, that the corresponding generating function $S_{1}^{*}$, which ought to be of the first order in the parameter $\sigma_{4} \equiv J_{4}+J_{2}^{2}$, must then satisfy

$$
\partial S_{1}^{*} / \partial \beta_{1}^{\prime}=\text { zeroth order in } \sigma_{4}
$$

One may alternatively eliminate short-periodic and long-periodic terms simultaneously, but one then obtains a Poisson term of the form $v^{\prime} \sin 2 \beta_{2}^{\prime}$ in $\alpha_{2}-\alpha_{2}^{\prime}$. Since $v^{\prime}$ has a secular part, such a result would appear absurd, since the "constant" $\alpha_{2}$, which ought to have only a small periodic variation, would then increase indefinitely with time.

These difficulties are examples of the failure of the von Zeipel method whenever the following conditions both hold: (1) the perturbing potential has a long-periodic part of the first order in the perturbation parameter, and (2) the canonical variables are such that the unperturbed Hamiltonian depends only on $L$.

To obtain a successful set of variables, we may proceed as follows. Let $q_{i}^{\mathbf{0}}$ and $p_{i}^{\mathbf{0}}, i=1,2,3$, be the coordinates and momenta $\rho, \eta, \phi, p_{\rho}, p_{\eta}, p_{\phi}$ corresponding to the unperturbed problem (the reference orbit), with Hamiltonian $F=F_{0}$. Also let $j_{i}^{0}, w_{i}^{0}, i=1,2,3$, be the corresponding action and angle variables.

Then

$$
\begin{aligned}
& p_{i}^{0}=\partial S\left(q_{1}^{0}, q_{2}^{0}, q_{3}^{0}, j_{1}^{0}, j_{2}^{0}, j_{3}^{0}\right) / \partial q_{i}^{0} \\
& w_{i}^{0}=\partial S\left(q_{1}^{0}, q_{2}^{0}, q_{3}^{0}, j_{1}^{0}, j_{2}^{0}, j_{3}^{0}\right) / \partial j_{i}^{0}, \quad(i=1,2,3)
\end{aligned}
$$

where $S$ is the Hamilton-Jacobi function of the unperturbed problem [Vinti 1959b], with the Jacobi $\alpha$ 's replaced by the $j_{i}^{0}$. Here

$$
j_{i}^{0} \equiv \oint p_{i}^{0} d q_{i}^{0}=j_{i}^{0}\left(\alpha_{1}, \alpha_{2}, \alpha_{3}\right)
$$

Now let $q_{i}, p_{i}, i=1,2,3$, be the coordinates and momenta corresponding to the perturbed problem, with Hamiltonian $F=F_{0}+F_{1}$. Introduce new variables $j_{i}, w_{i}, i=1,2,3$, by means of the canonical transformation

$$
\begin{aligned}
& p_{i}=\partial S\left(q_{1}, q_{2}, q_{3}, j_{1}, j_{2}, j_{3}\right) / \partial q_{i} \\
& w_{i}=\partial S\left(q_{1}, q_{2}, q_{3}, j_{1}, j_{2}, j_{3}\right) / \partial j_{i}, \quad(i=1,2,3)
\end{aligned}
$$

where $S$ is the same function of the $q_{i}$ and $j_{i}$ that the above Hamilton-Jacobi function is of the $q_{i}^{0}$ and $j_{i}^{0}$. Then the $w_{i}$ and $j_{i}$ are canonical variables, satisfying the equations

$$
\begin{gathered}
d j_{i} / d t=\partial F / \partial w_{i} \neq 0 \\
d w_{i} / d t=-\partial F / \partial j_{i} \neq \text { constant }
\end{gathered}
$$


They are thus not action and angle variables, since the $j_{i}$ are not constant and the $w_{i}$ are not linear functions of $t$. Moreover

$$
j_{i} \neq \oint p_{i} d q_{i}
$$

in contradistinction with (3.17).

It pays to go further, however, and introduce still another set of variables, a new Delaunay set $L, G, H, l, g, h$, by the transformations

$$
\begin{aligned}
& 2 \pi L=j_{1}+j_{2}+j_{3} \operatorname{sgn} \alpha_{3} \quad l=2 \pi w_{1} \\
& 2 \pi G=j_{2}+j_{3} \operatorname{sgn} \alpha_{3} \quad(3.23) \quad g=2 \pi\left(w_{2}-w_{1}\right) \\
& 2 \pi H=j_{3} \quad h=2 \pi\left(w_{3}-w_{2} \operatorname{sgn} \alpha_{3}\right),
\end{aligned}
$$

where $\operatorname{sgn} \alpha_{3}= \pm 1$ respectively for a direct orbit or a retrograde orbit. To verify that they are canonical, note that

$$
L d l+G d g+H d h=j_{1} d w_{1}+j_{2} d w_{2}+j_{3} d w_{3} .
$$

They were used by Izsak [1962] in his application of the author's reference orbit to the problem of the critical inclination. From (3.23) we now have

$$
j_{1}=2 \pi(L-G) \quad j_{2}=2 \pi\left(G-H \operatorname{sgn} \alpha_{3}\right) \quad j_{3}=2 \pi H .
$$

\section{The New Delaunay Set}

The functional relations among any of the quantities $\alpha_{i}, j_{i}, w_{i}$ for the perturbed problem are the same as those connecting $\alpha_{i}^{0}, j_{i}^{0}, w_{i}^{0}$ for the unperturbed problem. We may therefore usually drop superscript zeros and depend on the context for the meanings of the quantities.

From the author's paper [Vinti 1959 b] we now find

$$
\begin{aligned}
& j_{1}=2 \int_{\rho 1}^{\rho_{2}} \frac{\partial S}{\partial \rho} d \rho=2 \pi\left[\mu\left(-2 \alpha_{1}\right)^{-1 / 2}-\alpha_{2}\right]+\mathrm{O}\left(J_{2}\right) \\
& j_{2}=4 \int_{0}^{\eta} \frac{\partial S}{\partial \eta} d \eta=2 \pi\left(\alpha_{2}-\alpha_{3} \operatorname{sgn} \alpha_{3}\right)+\mathrm{O}\left(J_{2}\right) \\
& j_{3}=2 \pi \alpha_{3} .
\end{aligned}
$$

Next put

$$
\alpha_{r s} \equiv \partial \alpha_{r} / \partial j_{s} \quad(4.03) \quad \nu_{i} \equiv \partial \alpha_{1}^{0} / \partial j_{i}^{0} \quad(4.04) \quad j_{r s} \equiv \partial j_{r} / \partial \alpha_{s}
$$

Then, since

$$
\sum_{i=1}^{3} \frac{\partial S}{\partial j_{i}} \frac{\partial j_{i}}{\partial \alpha_{k}}=\frac{\partial S}{\partial \alpha_{k}}, \quad(k=1,2,3)
$$

we find

$$
\begin{aligned}
& \frac{\partial S}{\partial \alpha_{1}}=t+\beta_{1}=j_{11} w_{1}+j_{21} w_{2} \\
& \frac{\partial S}{\partial \alpha_{2}}=\beta_{22}=j_{12} w_{1}+j_{22} w_{2} \\
& \frac{\partial S}{\partial \alpha_{3}}=\beta_{3}=j_{13} w_{1}+j_{23} w_{2}+2 \pi w_{3} .
\end{aligned}
$$


With the aid of (3.24) these equations become

$$
\begin{aligned}
2 \pi\left(t+\beta_{1}\right) & =j_{11} l+j_{21}(l+g) \\
2 \pi \beta_{2} & =j_{12} l+j_{22}(l+g) \\
2 \pi \beta_{3} & =j_{13} l+\left(j_{23}+2 \pi \operatorname{sgn} \alpha_{3}\right)(l+g)+2 \pi h .
\end{aligned}
$$

The quantities $j_{r s}$ occurring here are given explicitly as functions of the $\alpha$ 's in eqs (7.16) through (7.21) of an earlier paper [Vinti 1961a].

The constant orbital elements in the perturbed problem are then the constant parts $a^{\prime \prime}, e^{\prime \prime}$, $\eta_{0}^{\prime \prime}$ of $a, e, \eta_{0}$, along with the initial values $l_{0}^{\prime \prime}, g_{0}^{\prime \prime}, h_{0}^{\prime \prime}$ of the secular parts of $l, g, h$. The corresponding Hamiltonian $F$ is given by

$$
F=F_{0}(L, G, H)+F_{1} \quad(4.13) \quad F_{0}=-\alpha_{1},
$$

so that

$$
\begin{array}{ll}
\dot{L}=\partial F / \partial l & \dot{l}=-\partial F / \partial L \\
\dot{G}=\partial F / \partial g \quad(4.15) & \dot{g}=-\partial F / \partial G \\
\dot{H}=\partial F / \partial h=0 . & \dot{h}=-\partial F / \partial H .
\end{array}
$$

With the use of (3.23), (3.26), and (4.04), we find for the unperturbed problem that

$$
\begin{aligned}
& \dot{l}=-\frac{\partial F_{0}}{\partial L}=\frac{\partial \alpha_{1}}{\partial L}=\sum_{i=1}^{3} \frac{\partial \alpha_{1}}{\partial j_{i}} \frac{\partial j_{i}}{\partial L}=2 \pi \nu_{1} \\
& \dot{g}=-\frac{\partial F_{0}}{\partial G}=\frac{\partial \alpha_{1}}{\partial G}=\sum_{i=1}^{3} \frac{\partial \alpha_{1}}{\partial j_{i}} \frac{\partial j_{i}}{\partial G}=2 \pi\left(\nu_{2}-\nu_{1}\right) \\
& \dot{h}=-\frac{\partial F_{0}}{\partial H}=\frac{\partial \alpha_{1}}{\partial H}=\sum_{i=1}^{3} \frac{\partial \alpha_{1}}{\partial j_{i}} \frac{\partial j_{i}}{\partial H}=2 \pi\left(\nu_{3}-\nu_{2} \operatorname{sgn} \alpha_{3}\right)
\end{aligned}
$$

and thus for the unperturbed problem that

$$
\begin{aligned}
& l=l_{0}+2 \pi \nu_{1} t \\
& g=g_{0}+2 \pi\left(\nu_{2}-\nu_{1}\right) t \\
& h=h_{0}+2 \pi\left(\nu_{3}-\nu_{2} \operatorname{sgn} \alpha_{3}\right) t,
\end{aligned}
$$

where we have dropped the double primes from $l_{0}, g_{0}, h_{0}$.

Before finding the effects of the perturbing potential, it is desirable to change the algorithm for the unperturbed problem, given in an earlier paper [Vinti 1961 a, pp. 197-200], so that the constant orbital elements become $a, e, I, l_{0}, g_{0}$, and $\beta_{3}$. ( $\beta_{3}$ is better than $h_{0}$, as we shall see later.) To do so, insert (4.10), (4.11), and (4.18) into eqs (8.2) and (8.3) of that paper and carry out the same process that was carried out there. One finds

$$
\begin{aligned}
M_{s} & =l_{0}+2 \pi \nu_{1} t \\
\psi_{s} & =l_{0}+g_{0}+2 \pi \nu_{2} t .
\end{aligned}
$$

With these new definitions of $M_{s}$ and $\psi_{s}$ the algorithm then becomes the same as in the earlier paper. Note, however, that the restriction on the angle of inclination $I$ has been removed [Vinti 1962].

\section{Variations in the Spheroidal Coordinates}

It is convenient to derive here the variations in $\rho, \eta$, and $\phi$ that will arise from the variations produced by the perturbing potential in the Delaunay variables. From the later solution of the 
canonical equations (4.15) and (4.16) we shall find that the $m$ th zonal harmonic produces variations in the Delaunay variables of the forms

$$
\begin{aligned}
& \delta L=\sigma_{m} L_{m} \quad \delta l=\sigma_{m} l_{m}+\sigma_{m} J_{2}^{-1} \tilde{l}_{m} \\
& \delta G=\sigma_{m} G_{m}+\sigma_{m} J_{2}^{-1} \tilde{G}_{m} \quad(5.00) \quad \delta g=\sigma_{m} g_{m}+\sigma_{m} J_{2}^{-1} \tilde{g}_{m} \\
& \delta H=0 \text {. } \\
& \delta h=\sigma_{m} h_{m}+\sigma_{m} J_{2}^{-1} \widetilde{h}_{m} \text {. }
\end{aligned}
$$

Here $\sigma_{3}=J_{3}$ and $\sigma_{4}=J_{4}+J_{2}^{2}$. The terms $\sigma_{m} L_{m}$ and $\sigma_{m} G_{m}$ are short-periodic of order $J_{2}^{2}$; the products of $\sigma_{m} J_{2}^{-1}$ with $\widetilde{G}_{m}, \widetilde{l}_{m}, \widetilde{g}_{m}$, and $\widetilde{h}_{m}$ are long-periodic of order $J_{2}$. For $m=3$ the products of $\sigma_{3}$ with $l_{3}, g_{3}$, and $h_{3}$ are short-periodic of order $J_{2}^{2}$. For $m=4$

$$
\begin{aligned}
& l_{4}=l_{41}+l_{42} \\
& g_{4}=g_{41}+g_{42} \\
& h_{4}=h_{41}+h_{42},
\end{aligned}
$$

where the products of $\sigma_{4}$ with $l_{41}, g_{41}$, and $h_{41}$ are short-periodic terms of order $J_{2}^{2}$ and where the products of $\sigma_{4}$ with $l_{42}, g_{42}$, and $h_{42}$ are secular terms of order $J_{2}^{2}$.

From $\delta L$ and $\delta G$ we can find the variations of the $j$ 's, then of the $\alpha$ 's, and finally of the elements $a, e$, and $\eta_{0} \equiv \sin I$. From these and from $\delta l, \delta g$, and $\delta h$ we can then find $\delta E, \delta v, \delta \psi$, and $\delta \chi$ and finally the coordinate variations $\delta \rho, \delta \eta$, and $\delta \phi$. To obtain the $\delta j$ 's, use (3.26) and (5.00). The results are

$$
\begin{aligned}
\delta j_{1} & =2 \pi\left(\sigma_{m} L_{m}-\sigma_{m} G_{m}-\sigma_{m} \tilde{G}_{m} / J_{2}\right) \\
\delta j_{2} & =2 \pi\left(\sigma_{m} G_{m}+\sigma_{m} \tilde{G}_{m} / J_{2}\right) \\
\delta j_{3} & =0 .
\end{aligned}
$$

To find the $\delta \alpha$ 's use

$$
\delta \alpha_{r}=\sum_{i=1}^{3} \frac{\partial \alpha_{r}}{\partial j_{i}} \delta j_{i}
$$

Within the accuracy of the calculation, the coefficients $\partial \alpha_{r} / \partial j_{i}$ are needed only through order $J_{2}^{0}$. Thus by (5.03), (5.04), and (4.04)

$$
\delta \alpha_{1}=2 \pi \nu_{1} \sigma_{m} L_{m}+2 \pi\left(\nu_{2}-\nu_{1}\right) \sigma_{m} G_{m}+2 \pi\left(\nu_{2}-\nu_{1}\right) \sigma_{m} \tilde{G}_{m} / J_{2} .
$$

Then, by (5.03), (5.04), and (4.03)

$$
\delta \alpha_{2}=2 \pi \alpha_{21} \sigma_{m} L_{m}+2 \pi\left(\alpha_{22}-\alpha_{21}\right) \sigma_{m} G_{m}+2 \pi\left(\alpha_{22}-\alpha_{21}\right) \sigma_{m} \tilde{G}_{m} / J_{2}
$$

Also, by (4.02) and (5.03)

$$
\delta \alpha_{3}=0
$$

To find $\alpha_{21}$ and $\alpha_{22}$ note that

so that

$$
\begin{aligned}
& \sum_{i=1}^{3} \frac{\partial \alpha_{2}}{\partial j_{i}} \frac{\partial j_{i}}{\partial \alpha_{2}}=\frac{\partial \alpha_{2}}{\partial \alpha_{2}}=1 \\
& \sum_{i=1}^{3} \frac{\partial \alpha_{2}}{\partial j_{i}} \frac{\partial j_{i}}{\partial \alpha_{1}}=\frac{\partial \alpha_{2}}{\partial \alpha_{1}}=0
\end{aligned}
$$

$$
\begin{aligned}
& \alpha_{21} j_{21}+\alpha_{22} j_{22}=1 \\
& \alpha_{21} j_{11}+\alpha_{22} j_{12}=0
\end{aligned}
$$


with the solution

where

$$
\alpha_{21}=-j_{21} / \Delta \quad \alpha_{22}=j_{11} / \Delta
$$

$$
\Delta \equiv j_{11} j_{22}-j_{12} j_{21} .
$$

From an earlier paper [Vinti 1961 a, p 189], it then follows that

and also that

$$
\alpha_{21}=\mathrm{O}\left(J_{2}\right) \quad \alpha_{22}=(2 \pi)^{-1}+\mathrm{O}\left(J_{2}\right)
$$

where

$$
\begin{aligned}
2 \pi \nu_{1} & =n+\mathrm{O}\left(J_{2}\right) \\
\nu_{2}-\nu_{1} & =\mathrm{O}\left(J_{2}\right),
\end{aligned}
$$

On carrying secular and short-periodic variations only through order $J_{2}^{2}$ and long-periodic perturbations only through order $J_{2}$, it then follows from (5.04) through (5.08) and (5.12) through (5.14) that

$$
\begin{aligned}
& \delta \alpha_{1}=n \sigma_{m} L_{m} \\
& \delta \alpha_{2}=\sigma_{m} G_{m}+\sigma_{m} \tilde{G}_{m} / J_{2} \\
& \delta \alpha_{3}=0 .
\end{aligned}
$$

In finding the variations of other quantities we may drop all terms in their defining equations of order $J_{2}^{k}$, where $k \geqq 1$. To show this, note that if $P=\mathrm{O}\left(J_{2}^{0}\right)$ and $\delta P=\sigma_{m} P_{1}+\sigma_{m} J_{2}^{-1} \widetilde{P}_{1}$ where $P_{1}$ and $\tilde{P}_{1}$ are both of order $J_{2}^{0}$, then

$$
\delta\left(J_{2}^{k} P\right)=J_{2}^{k}\left(\sigma_{m} P_{1}+\sigma_{m} J_{2}^{-1} \tilde{P}_{1}\right)
$$

Thus if $k \geqq 1, J_{2}^{k} \sigma_{m} P_{1}$ is a secular plus short-periodic term of order $k+2 \geqq 3$ and $J_{2}^{k} \sigma_{m} J_{2}^{-1} \tilde{P}_{1}$ is a long-periodic term of order $k+1 \geqq 2$. This proves the statement.

To obtain the variations of $a, e$, and $\eta_{0}$ we may thus use

$$
a \approx-\frac{\mu}{2 \alpha_{1}} \quad 1-e^{2} \approx-\frac{2 \alpha_{1} \alpha_{2}^{2}}{\mu^{2}} \quad 1-\eta_{0}^{2} \approx \frac{\alpha_{3}^{2}}{\alpha_{2}^{2}}
$$

the neglected terms being of order $J_{2}$. There follow

$$
\begin{gathered}
\delta a=\frac{\mu}{2 \alpha_{1}^{2}} \delta \alpha_{1}=\frac{2 a^{2}}{\mu} \delta \alpha_{1} \\
e \delta e=\frac{\alpha_{2}^{2}}{\mu^{2}} \delta \alpha_{1}+\frac{2 \alpha_{1} \alpha_{2}}{\mu^{2}} \delta \alpha_{2} \\
\eta_{0} \delta \eta_{0}=\frac{\alpha_{3}^{2}}{\alpha_{2}^{3}} \delta \alpha_{2} .
\end{gathered}
$$

With use of (5.15), (5.16), (5.19), (5.20) and the relation

$$
\begin{aligned}
\alpha_{2}^{2} & =\mu p+\mathrm{O}\left(J_{2}\right), \text { we then find } \\
\delta a & =\frac{2 \sigma_{m} L_{m}}{a n} \\
\delta e & =\frac{p n}{\mu e} \sigma_{m} L_{m}-(a e)^{-1}\left(\frac{p}{\mu}\right)^{1 / 2}\left(\sigma_{m} G_{m}+\sigma_{m} J_{2}^{-1} \tilde{G}_{m}\right) \\
\delta \eta_{0} & =\eta_{0}^{-1}(\mu p)^{-1 / 2}\left(1-\eta_{0}^{2}\right)\left(\sigma_{m} G_{m}+\sigma_{m} J_{2}^{-1} \tilde{G}_{m}\right) .
\end{aligned}
$$


The element $a$ thus has only a short-periodic variation while the elements $e$ and $\eta_{0} \equiv \sin I$ have both short-periodic and long-periodic but no secular variations.

To find $\delta E, \delta v$, and $\delta \psi$ we first insert (4.10) and (4.11) into eqs (8.2) and (8.3) of the earlier paper [Vinti 1961 a], rejecting all terms of order $J_{2}^{k}$, where $k \geqq 1$. We find

$$
\begin{aligned}
E-e \sin E & =l+\mathrm{O}\left(J_{2}\right) \\
\psi & =v+g+\mathrm{O}\left(J_{2}\right)
\end{aligned}
$$

Equation (5.24) gives

$$
\delta E=(1-e \cos E)^{-1}(\delta l+\sin E \delta e) .
$$

To find $\delta v$, we use the anomaly connection

$$
\tan \frac{v}{2}=\left(\frac{1+e}{1-e}\right)^{1 / 2} \tan \frac{E}{2}
$$

from which there follows

$$
\begin{aligned}
\delta v & =\left(\frac{1+e}{1-e}\right)^{1 / 2}\left(1-\frac{2 e}{1+e} \sin ^{2} \frac{v}{2}\right) \delta E+\frac{\sin \imath}{1-e^{2}} \delta e \\
& =\left(1-e^{2}\right)^{1 / 2}(1-e \cos E)^{-1}\left[\delta E+\left(1-e^{2}\right)^{-1} \sin E \delta e\right] .
\end{aligned}
$$

Then

$$
\delta \psi=\delta v+\delta g .
$$

The variations of the spheroidal coordinates

$$
\begin{aligned}
& \rho=a(1-e \cos E) \\
& \eta=\eta_{0} \sin \psi
\end{aligned}
$$

are then

$$
\begin{aligned}
& \delta \rho=(1-e \cos E) \delta a-a \cos E \delta e+a e \sin E \delta E \\
& \delta \eta=\sin \psi \delta \eta_{0}+\eta_{0} \cos \psi \delta \psi
\end{aligned}
$$

To find the variation $\delta \phi$ of the right ascension, we note that by eq (8.49) of the earlier paper [Vinti 1961 a],

$$
\phi=\beta_{3}+\chi \operatorname{sgn} \alpha_{3}+\mathrm{O}\left(J_{2}\right)
$$

where

$$
\begin{aligned}
& \tan \chi=\left(1-\eta_{0}^{2}\right)^{1 / 2} \tan \psi \\
& \cos \chi=\left(1-\eta_{0}^{2} \sin ^{2} \psi\right)^{-1 / 2} \cos \psi .
\end{aligned}
$$

Equation (5.36) was given in Vinti [1961 b]. Also by (4.12) and the results

$$
j_{13}=\mathrm{O}\left(J_{2}\right) \quad j_{13}+2 \pi \operatorname{sgn} \alpha_{3}=\mathrm{O}\left(J_{2}\right)
$$


from page 189 of Vinti [1961 a], we find

$$
\beta_{3}=h+\mathrm{O}\left(J_{2}\right)
$$

so that

$$
\delta \beta_{3}=\delta h
$$

From (5.35) and (5.36) we find

$$
\delta \chi=\left(1-\eta_{0}^{2} \sin ^{2} \psi\right)^{-1}\left[\left(1-\eta_{0}^{2}\right)^{1 / 2} \delta \psi-\frac{1}{2}\left(1-\eta_{0}^{2}\right)^{-1 / 2} \eta_{0} \sin 2 \psi \delta \eta_{0}\right] .
$$

Then, from (5.34), (5.39), (5.40), and (5.23), it follows that

$$
\delta \phi=\delta h+\left(1-\eta_{0}^{2} \sin ^{2} \psi\right)^{-1} \cos I\left[\delta \psi-\frac{1}{2}(\mu p)^{-1 / 2} \sin 2 \psi\left(\sigma_{m} G_{m}+\sigma_{m} J_{2}^{-1} \tilde{G}_{m}\right)\right],
$$

where we have used $\left(1-\eta_{0}^{2}\right)^{1 / 2} \operatorname{sgn} \alpha_{3}=\cos I$.

It is well to note here that $\beta_{3}$ is just as useful an orbital element as $h_{0}$.

\section{Solution for the Perturbed Delaunay Variables}

We now have to solve the canonical equations (4.13) through (4.16), the perturbing potential being

$$
F_{1}=-\mu r e^{4} r^{-5} \sigma_{4} P_{4}(\sin \theta)
$$

in the case of the residual fourth harmonic. To obtain secular and short-periodic variations through order $J_{2}^{2}$ and long-periodic variations through order $J_{2}$, it will suffice to use elliptic approximations in (6.00), since $\sigma_{4} \equiv J_{4}+J_{2}^{2}=\mathrm{O}\left(J_{2}^{2}\right)$. Thus in (6.00) we may put

$$
\begin{gathered}
\sin \theta=\eta=\eta_{0} \sin \psi=\eta_{0} \sin (v+g) \\
r=\rho=a(1-e \cos E)=a\left(1-e^{2}\right)(1+e \cos v)^{-1}
\end{gathered}
$$

and we may use

$$
\begin{aligned}
l & =E-e \sin E \\
d l & =(r / a)^{2}\left(1-e^{2}\right)^{-1 / 2} d v \\
\psi & =v+g .
\end{aligned}
$$

Then

$$
F_{1}=\frac{-\mu r_{e}^{4} \sigma_{4}}{8 a^{5}}\left[\left(3-15 \eta_{0}^{2}+\frac{105}{8} \eta_{0}^{4}\right)\left(\frac{a}{r}\right)^{5}+5 \eta_{0}^{2}\left(3-\frac{7}{2} \eta_{0}^{2}\right)\left(\frac{a}{r}\right)^{5} \cos 2 \psi+\frac{35}{8} \eta_{0}^{4}\left(\frac{a}{r}\right)^{5} \cos 4 \psi\right] .
$$

The part of $F_{1}$ independent of $l$ is then

$$
\begin{aligned}
F_{1 m} \equiv(2 \pi)^{-1} \int_{0}^{2 \pi} F_{1} d l=-\frac{\mu r_{e}^{4} \sigma_{4}}{16 \pi a^{5}}\left(1-e^{2}\right)^{-1 / 2} \int_{0}^{2 \pi} & {\left[\left(3-15 \eta_{0}^{2}+\frac{105}{8} \eta_{0}^{4}\right)\left(\frac{a}{r}\right)^{3}\right.} \\
& \left.+5 \eta_{0}^{2}\left(3-\frac{7}{2} \eta_{0}^{2}\right)\left(\frac{a}{r}\right)^{3} \cos 2 \psi+\frac{35}{8} \eta_{0}^{4}\left(\frac{a}{r}\right)^{3} \cos 2 \psi\right] d v
\end{aligned}
$$


From (6.02) and (6.05) it follows that

$$
\begin{gathered}
\int_{0}^{2 \pi}\left(\frac{a}{r}\right)^{3} d v=2 \pi\left(1-e^{2}\right)^{-3}\left(1+\frac{3}{2} e^{2}\right) \\
\int_{0}^{2 \pi}\left(\frac{a}{r}\right)^{3} \cos 2 \psi d v=\frac{3}{2} \pi e^{2}\left(1-e^{2}\right)^{-3} \cos 2 g \\
\int_{0}^{2 \pi}\left(\frac{a}{r}\right)^{3} \cos 4 \psi d v=0 .
\end{gathered}
$$

Equations (6.07) through (6.10) then result in

$$
F_{1 m}=F_{1 c}+F_{1 p}
$$

where the constant part is

$$
F_{1 c}=-\frac{\mu r_{e}^{4} \sigma_{4}}{8 a^{5}}\left(1-e^{2}\right)^{-7 / 2}\left(1+\frac{3}{2} e^{2}\right)\left(3-15 \eta_{0}^{2}+\frac{105}{8} \eta_{0}^{4}\right)
$$

and the long-periodic part is

$$
F_{1 p}=-\frac{15 \mu r_{e}^{4} \sigma_{4}}{32 a^{5}}\left(1-e^{2}\right)^{-7 / 2} e^{2} \eta_{0}^{2}\left(3-\frac{7}{2} \eta_{0}^{2}\right) \cos 2 g
$$

The short-periodic part is then given by

$$
F_{1 l}=F_{1}-F_{1 c}-F_{l p},
$$

so that

$$
\begin{aligned}
F_{1 l}=-\frac{\mu r_{e}^{4} \sigma_{4}}{8 a^{5}} & \left\{\left(3-15 \eta_{0}^{2}+\frac{105}{8} \eta_{0}^{4}\right)\left[\left(\frac{a}{r}\right)^{5}-\left(1-e^{2}\right)^{-7 / 2}\left(1+\frac{3}{2} e^{2}\right)\right]\right. \\
+ & \left.5 \eta_{0}^{2}\left(3-\frac{7}{2} \eta_{0}^{2}\right)\left[\left(\frac{a}{r}\right)^{5} \cos 2 \psi-\frac{3}{4} e^{2}\left(1-e^{2}\right)^{-7 / 2} \cos 2 g\right]+\frac{35}{8} \eta_{0}^{4}\left(\frac{a}{r}\right)^{5} \cos 4 \psi\right\} .
\end{aligned}
$$

From (3.23) and (4.00) through (4.02) we now obtain

$$
L=\mu\left(-2 \alpha_{1}\right)^{-1 / 2} \quad G=\alpha_{2} \quad H=\alpha_{3},
$$

with neglect of terms of order $J_{2}$. To the same accuracy

$$
a=-\frac{1}{2} \mu \alpha_{1}^{-1} \quad \alpha_{2}^{2}=\mu a\left(1-e^{2}\right) \quad \alpha_{3}^{2} \alpha_{2}^{-2}=1-\eta_{0}^{2} .
$$

Thus, with neglect of terms of order $J_{2}$,

$$
a=L^{2} \mu^{-1} \quad 1-e^{2}=G^{2} L^{-2} \quad \eta_{0}^{2}=1-H^{2} G^{-2},
$$

as expected. When we later take derivatives of a generating function with respect to $L, G$, and $H$, we shall have to use the expressions (6.18) to replace them in the results by the elements $a, e$, and $\eta_{0}$.

In solving the canonical equations (4.15) and (4.16) we first make a canonical transformation to new canonical variables $L^{\prime}, G^{\prime}, H^{\prime}, l^{\prime}, g^{\prime}$, and $h^{\prime}$, so that the new Hamiltonian $F^{*}$ shall be independent of $l^{\prime}$ and $h^{\prime}$. This first step will yield the short-periodic effects. To carry it out, introduce the generating function

$$
S\left(L^{\prime}, G^{\prime}, H^{\prime}, l, g, h\right)=S_{0}+S_{1}\left(L^{\prime}, G^{\prime}, H^{\prime}, l, g\right),
$$


where

$$
S_{0} \equiv L^{\prime} l+G^{\prime} g+H^{\prime} h
$$

and where $S_{1}$ is to be of the first order in $\sigma_{4}$. Then follow the von Zeipel method, as applied by Brouwer (1959) to satellite orbits.

On splitting $F^{*}$ into parts $F_{0}^{*}$ and $F_{1}^{*}$, we then have

$$
F_{0}(L, G, H)+F_{1}(L, G, H, l, g)=F_{0}^{*}\left(L^{\prime}, G^{\prime}, H^{\prime}\right)+F_{1}^{*}\left(L^{\prime}, G^{\prime}, H^{\prime}, g^{\prime}\right)
$$

along with the following relations connecting $S_{1}$ and the primed and unprimed variables:

$$
\begin{aligned}
& L=\frac{\partial S}{\partial l}=L^{\prime}+\frac{\partial S_{1}}{\partial l} \quad l^{\prime}=\frac{\partial S}{\partial L^{\prime}}=l+\frac{\partial S_{1}}{\partial L^{\prime}} \\
& G=\frac{\partial S}{\partial g}=G^{\prime}+\frac{\partial S_{1}}{\partial g} \quad \text { (6.22) } \quad g^{\prime}=\frac{\partial S}{\partial G^{\prime}}=g+\frac{\partial S_{1}}{\partial G^{\prime}} \\
& H=\frac{\partial S}{\partial h}=H^{\prime} \quad h^{\prime}=\frac{\partial S}{\partial H^{\prime}}=h+\frac{\partial S_{1}}{\partial H}
\end{aligned}
$$

Since the new Hamiltonian will not contain $l^{\prime}$ or $h^{\prime}$, we shall have $H^{\prime}=H=$ constant and $L^{\prime}=$ constant. Insertion of (6.22) and (6.23) into (6.21) then gives a partial differential equation for $S_{1}$ :

$$
\begin{aligned}
F_{0}\left(L^{\prime}+\frac{\partial S_{1}}{\partial l}, G^{\prime}+\frac{\partial S_{1}}{\partial g}, H\right)+F_{1}\left(L^{\prime}+\frac{\partial S_{1}}{\partial l}, G^{\prime}+\frac{\partial S_{1}}{\partial g},\right. & \left.H, l+\frac{\partial S_{1}}{\partial L^{\prime}}, g+\frac{\partial S_{1}}{\partial G^{\prime}}\right) \\
& =F_{0}^{*}\left(L^{\prime}, G^{\prime}, H\right)+F_{1}^{*}\left(L^{\prime}, G^{\prime}, H, g+\frac{\partial S_{1}}{\partial G^{\prime}}\right) .
\end{aligned}
$$

Taylor expansion in the neighborhood of $L^{\prime}, G^{\prime}, H, l$, and $g$, with rejection of terms beyond the first order in $\sigma_{4}$, then gives

$$
F_{0}\left(L^{\prime}, G^{\prime}, H\right)+\frac{\partial F_{0}}{\partial L^{\prime}} \frac{\partial S_{1}}{\partial l}+\frac{\partial F_{0}}{\partial G^{\prime}} \frac{\partial S_{1}}{\partial g}+F_{1}\left(L^{\prime}, G^{\prime}, H, l, g\right)=F_{0}^{*}\left(L^{\prime}, G^{\prime}, H\right)+F_{1}^{*}\left(L^{\prime}, G^{\prime}, H, g\right) .
$$

The zeroth order terms lead to

$$
F_{0}\left(L^{\prime}, G^{\prime}, H\right)=F_{0}^{*}\left(L^{\prime}, G^{\prime}, H\right)
$$

and the first order terms to

$$
\frac{\partial F_{0}}{\partial L^{\prime}} \frac{\partial S_{1}}{\partial l}+\frac{\partial F_{0}}{\partial G^{\prime}} \frac{\partial S_{1}}{\partial G^{\prime}}+F_{1 c}\left(L^{\prime}, G^{\prime}, H\right)+F_{1 p}\left(L^{\prime}, G^{\prime}, H, g\right)+F_{1 l}\left(L^{\prime}, G^{\prime}, H, l, g\right)=F_{1}^{*}\left(L^{\prime}, G^{\prime}, H, g^{\prime}\right)
$$

In writing down (6.27) we have used (6.14) to express $F_{1}$ as a sum of the terms $F_{1 c}, F_{1 p}$, and $F_{1 l}$ and we have replaced $g$ by $g^{\prime}$ in $F_{1}^{*}$, a permissible step involving an error of order $\sigma_{4}^{2}$. In (6.27) the terms independent of $l$ then yield

$$
F_{1}^{*}=F_{1 c}\left(L^{\prime}, G^{\prime}, H\right)+F_{1 p}\left(L^{\prime}, G^{\prime}, H, g\right)
$$

so that $(6.26)$ and (6.28) together provide the new Hamiltonian $F^{*}$. The remaining terms, depending on $l$, then yield

$$
\frac{\partial F_{0}}{\partial L^{\prime}} \frac{\partial S_{1}}{\partial l}+\frac{\partial F_{0}}{\partial G^{\prime}} \frac{\partial S_{1}}{\partial g}=-F_{1 l}\left(L^{\prime}, G^{\prime}, H, l, g\right)
$$


a partial differential equation for $S_{1}$. With use of (4.17) it becomes

$$
2 \pi \nu_{1} \frac{\partial S_{1}}{\partial l}+2 \pi\left(\nu_{2}-\nu_{1}\right) \frac{\partial S_{1}}{\partial g}=F_{1 l}\left(L^{\prime}, G^{\prime}, H, l, g\right)
$$

Since $\nu_{2}-\nu_{1}$ is of order $J_{2}$ and since $S_{1}$ is to be of order $\sigma_{4} \equiv J_{4}+J_{2}^{2}$, it follows that $2 \pi\left(\nu_{2}-\nu_{1}\right) \partial S_{1} / \partial g$ will be of order $J_{2}^{3}$ and is thus to be rejected. Thus

$$
2 \pi \nu_{1} S_{1}=\int F_{1 l}\left(L^{\prime}, G^{\prime}, H, l, g\right) d l+\Phi(g) .
$$

To evaluate the integral in (6.31), apply (6.15), (6.02), (6.04), and (6.18). Since $F_{1 l}$ has a factor $\sigma_{4}=O\left(J_{2}^{2}\right)$, we can make a number of approximations at this point and still achieve our desired accuracy. These are: drop the primes from $L^{\prime}$ and $G^{\prime}$ in (6.31), place

$$
2 \pi \nu_{1}=n \equiv \mu^{1 / 2} a^{-3 / 2},
$$

express $a, e$, and $\eta_{0}$ by means of (6.18), drop the primes from $L^{\prime}$ and $G^{\prime}$ in calculating $\partial S_{1} / \partial L^{\prime}$ and $\partial S_{1} / \partial G^{\prime}$, and finally use (6.18) again to replace $L, G$, and $H$ in the final formulas by $a$, e, and $\eta_{0}$.

We obtain

$$
\begin{aligned}
S_{1}=\sigma_{4} Q_{1} & {\left[\left(1+\frac{3}{2} e^{2}\right)(v-l)+\left(3 e+\frac{3}{4} e^{3}\right) \sin v+\frac{3}{4} e^{2} \sin 2 v+\frac{e^{3}}{12} \sin 3 v\right] } \\
+ & \sigma_{4} Q_{2}\left[\frac{3}{4} e^{2}(v-l) \cos 2 g+\frac{e^{3}}{8} \sin (v-2 g)+\left(\frac{3}{2} e+\frac{3}{8} e^{3}\right) \sin (v+2 g)\right. \\
+ & \left.\left(\frac{1}{2}+\frac{3}{4} e^{2}\right) \sin (2 v+2 g)+\left(\frac{e}{2}+\frac{e^{3}}{8}\right) \sin (3 v+2 g)+\frac{3 e^{2}}{16} \sin (4 v+2 g)+\frac{e^{3}}{40} \sin (5 v+2 g)\right] \\
& +\sigma_{4} Q_{3}\left[\frac{e^{3}}{8} \sin (v+4 g)+\frac{3}{8} e^{2} \sin (2 v+4 g)+\left(\frac{e}{2}+\frac{e^{3}}{8}\right) \sin (3 v+4 g)\right. \\
+\left(\frac{1}{4}+\right. & \left.\left.\frac{3}{8} e^{2}\right) \sin (4 v+4 g)+\left(\frac{3 e}{10}+\frac{3 e^{3}}{40}\right) \sin (5 v+4 g)+\frac{e^{2}}{8} \sin (6 v+4 g)+\frac{e^{3}}{56} \sin (7 v+4 g)\right]+\Phi(g),
\end{aligned}
$$

where $\Phi(g)$ is a constant of integration. Here

$$
Q_{i}=\frac{-\mu r_{e}^{4}}{8 n a^{5}}\left(\frac{L}{G}\right)^{7} q_{i}=-\frac{1}{8}\left(\frac{r_{e}}{p}\right)^{4}(\mu p)^{1 / 2} q_{i}
$$

where

$$
q_{1} \equiv 3-15 \eta_{0}^{2}+\frac{105}{8} \eta_{0}^{4} \quad q_{2} \equiv 5 \eta_{0}^{2}\left(3-\frac{7}{2} \eta_{0}^{2}\right) \quad q_{3} \equiv \frac{35}{8} \eta_{0}^{4}
$$

With use of (6.18) and (6.33) we find

$$
\frac{\mu L^{7}}{n a^{5}}=\mu^{4}+\mathrm{O}\left(J_{2}\right)
$$

and

$$
\begin{aligned}
Q_{1} & =\frac{-\mu^{4} r_{e}^{4}}{64 G^{7}}\left(9-90 \frac{H^{2}}{G^{2}}+105 \frac{H^{4}}{G^{4}}\right) \\
Q_{2} & =\frac{5 \mu^{4} r_{e}^{4}}{16 G^{7}}\left(1-\frac{H^{2}}{G^{2}}\right)\left(1-7 \frac{H^{2}}{G^{2}}\right) \\
Q_{3} & =\frac{-35 \mu^{4} r_{e}^{4}}{64 G^{7}}\left(1-\frac{H^{2}}{G^{2}}\right)^{2},
\end{aligned}
$$

through terms of order $J_{2}^{0}$.

To make $S_{1}$ purely short-periodic, one would have to choose $\Phi(g)$ in such a way that 
$2 \pi \bar{S}_{1} \equiv \int_{0}^{2 \pi} S_{1} d l$ would vanish. It turns out that $\Phi(g)$ would then not vanish, but would have to be a long-periodic term, just cancelling a long-periodic term of order $J_{2}^{2}$ arising from the rest of the expression for $S_{1}$. The later calculation of long-periodic effects, however, will be accurate only through order $J_{2}$. If we arbitrarily drop $\Phi(g)$, whose calculation would be extremely laborious, the net effect will be only to leave in the short-periodic terms of $G, l, g$, and $h$ some long-periodic impurities of order $J_{2}^{2}$, not affecting the accuracy of the calculation.

The errors of the short-periodic terms will be of order $J_{2}^{3}$, for two reasons. First, the shortperiodic terms of the reference orbit were calculated only through terms of order $J_{2}^{2}$; second, the present calculation makes use of elliptic approximations, so that a variation of the form $\sigma_{4} f$ has an error of order $J_{2}$ in $f$ and thus of order $J_{2}^{3}$ in $\sigma_{4} f$. For this second reason the secular corrections produced by the perturbing potential will also have errors of order $J_{2}^{3}$, even though the first omitted term in the von Zeipel equation (6.25) is of order $\sigma_{4}^{2}$ or $J_{2}^{4}$.

\section{Short-Periodic Terms}

It is now straightforward but tedious to calculate the short-periodic terms. From (6.22), (6.31), (6.32), and (6.15), we find, through terms of order $J_{2}^{2}$

$$
\frac{\partial S_{1}}{\partial l}=L-L^{\prime}=\sigma_{4} L_{4}
$$

where

$$
\begin{aligned}
L_{4}= & -\frac{r_{e}^{4}}{8 a^{5} n}\left\{\left(3-15 \eta_{0}^{2}+\frac{105}{8} \eta_{0}^{4}\right)\left[\left(\frac{a}{r}\right)^{5}-\left(1-e^{2}\right)^{-7 / 2}\left(1+\frac{3}{2} e^{2}\right)\right]\right. \\
& \left.+5 \eta_{0}^{2}\left(3-\frac{7}{2} \eta_{0}^{2}\right)\left[\left(\frac{a}{r}\right)^{5} \cos (2 v+2 g)-\frac{3 e^{2}}{4}\left(1-e^{2}\right)^{-7 / 2} \cos 2 g\right]+\frac{35}{8} \eta_{0}^{4}\left(\frac{a}{r}\right)^{5} \cos (4 v+4 g)\right\} .
\end{aligned}
$$

From (6.22) and (6.33), we find

$$
\frac{\partial S_{1}}{\partial g}=G-G^{\prime}=\sigma_{4} G_{4}
$$

where

$$
\begin{aligned}
G_{4}= & Q_{2}\left[-\frac{3}{2} e^{2}(v-l) \sin 2 g-\frac{e^{3}}{4} \cos (v-2 g)+\left(3 e+\frac{3 e^{3}}{4}\right) \cos (v+2 g)\right. \\
& \left.+\left(1+\frac{3}{2} e^{2}\right) \cos (2 v+2 g)+\left(e+\frac{e^{3}}{4}\right) \cos (3 v+2 g)+\frac{3 e^{2}}{8} \cos (4 v+2 g)+\frac{e^{3}}{20} \cos (5 v+2 g)\right] \\
& +Q_{3}\left[\frac{1}{2} e^{3} \cos (v+4 g)+\frac{3}{2} e^{2} \cos (2 v+4 g)+\left(2 e+\frac{1}{2} e^{3}\right) \cos (3 v+4 g)+\left(1+\frac{3}{2} e^{2}\right) \cos (4 v+4 g)\right. \\
& \left.+\left(\frac{6 e}{5}+\frac{3 e^{3}}{10}\right) \cos (5 v+4 g)+\frac{1}{2} e^{2} \cos (6 v+4 g)+\frac{e^{3}}{14} \cos (7 v+4 g)\right] .
\end{aligned}
$$

Since the dependence of $S_{1}$ on $H$ is only through the $Q$ 's, the calculation of

$$
h-h^{\prime}=-\frac{\partial S_{1}}{\partial H}=\sigma_{4} h_{41}
$$

is simple. First calculate the derivatives $\partial Q_{i} / \partial H$. From (6.37) through (6.39) and the sufficiently 
accurate relations $H / G=\cos I, H^{2} / G^{2}=1-\eta_{0}^{2}$ and $G^{2}=\mu p$, we find

$$
\begin{aligned}
& \frac{\partial Q_{1}}{\partial H}=\frac{15}{16}\left(\frac{r_{e}}{p}\right)^{4}\left(7 \eta_{0}^{2}-4\right) \cos I \\
& \frac{\partial Q_{2}}{\partial H}=\frac{5}{4}\left(\frac{r_{e}}{p}\right)^{4}\left(3-7 \eta_{0}^{2}\right) \cos I \\
& \frac{\partial Q_{3}}{\partial H}=\frac{35}{16}\left(\frac{r_{e}}{p}\right)^{4} \eta_{0}^{2} \cos I .
\end{aligned}
$$

From (7.04), (6.33), and (7.05) we then obtain

$$
\begin{aligned}
h_{41}=- & \frac{5}{16}\left(\frac{r_{e}}{p}\right)^{4} \cos I\left\{3\left(7 \eta_{0}^{2}-4\right)\left[\left(1+\frac{3}{2} e^{2}\right)(v-l)+\left(3 e+\frac{3 e^{3}}{4}\right) \sin v+\frac{3 e^{2}}{4} \sin 2 v+\frac{e^{3}}{12} \sin 3 v\right]\right. \\
+ & 4(3-7 \eta \text { g })\left[\frac{3}{4} e^{2}(v-l) \cos 2 g+\frac{e^{3}}{8} \sin (v-2 g)+\left(\frac{3 e}{2}+\frac{3 e^{3}}{8}\right) \sin (v+2 g)\right. \\
+ & \left.\left(\frac{1}{2}+\frac{3 e^{2}}{4}\right) \sin (2 v+2 g)+\left(\frac{e}{2}+\frac{e^{3}}{8}\right) \sin (3 v+2 g)+\frac{3 e^{2}}{16} \sin (4 v+2 g)+\frac{e^{3}}{40} \sin (5 v+2 g)\right] \\
+ & 7 \eta_{0}^{2}\left[\frac{e^{3}}{8} \sin (v+4 g)+\frac{3 e^{2}}{8} \sin (2 v+4 g)+\left(\frac{e}{2}+\frac{e^{3}}{8}\right) \sin (3 v+4 g)+\left(\frac{1}{4}+\frac{3 e^{2}}{8}\right) \sin (4 v+4 g)\right. \\
& \left.\left.+\left(\frac{3 e}{10}+\frac{3 e^{3}}{40}\right) \sin (5 v+4 g)+\frac{e^{2}}{8} \sin (6 v+4 g)+\frac{e^{3}}{56} \sin (7 v+4 g)\right]\right\} .
\end{aligned}
$$

To find $l-l^{\prime}$ and $g-g^{\prime}$, we use (6.23), (6.33), and (6.38) through (6.40). We may write (6.33) as

$$
S_{1}=\sigma_{4} Q_{1}(G, H) f_{1}(e, v)+\sigma_{4} Q_{2}(G, H) f_{2}(e, v)+\sigma_{4} Q_{3}(G, H) f_{3}(e, v),
$$

where

$$
f_{1}(e, v) \equiv\left(1+\frac{3}{2} e^{2}\right)(v-l)+\left(3 e+\frac{3 e^{3}}{4}\right) \sin v+\frac{3}{4} e^{2} \sin 2 v+\frac{e^{3}}{12} \sin 3 v
$$

$f_{2}(e, v) \equiv \frac{3}{4} e^{2}(v-l) \cos 2 g+\frac{e^{3}}{8} \sin (v-2 g)+\left(\frac{3 e}{2}+\frac{3 e^{3}}{8}\right) \sin (v+2 g)$

$$
+\left(\frac{1}{2}+\frac{3}{4} e^{2}\right) \sin (2 v+2 g)+\left(\frac{e}{2}+\frac{e^{3}}{8}\right) \sin (3 v+2 g)+\frac{3 e^{2}}{16} \sin (4 v+2 g)+\frac{e^{3}}{40} \sin (5 v+2 g)
$$

$f_{3}(e, v) \equiv \frac{e^{3}}{8} \sin (v+4 g)+\frac{3 e^{2}}{8} \sin (2 v+2 g)+\left(\frac{e}{2}+\frac{e^{3}}{8}\right) \sin (3 v+4 g)$

$$
+\left(\frac{1}{4}+\frac{3 e^{2}}{8}\right) \sin (4 v+4 g)+\left(\frac{3 e}{10}+\frac{3 e^{3}}{40}\right) \sin (5 v+4 g)+\frac{e^{2}}{8} \sin (6 v+4 g)+\frac{e^{3}}{56} \sin (7 v+4 g) .
$$

Then

$$
\begin{aligned}
\frac{\partial S_{1}}{\partial L} & =\sigma_{4} \sum_{i=1}^{3} Q_{i}\left(\frac{\partial f_{i}}{\partial e}+\frac{\partial f_{i}}{\partial v} \frac{\partial v}{\partial e}\right) \frac{\partial e}{\partial L} \\
\frac{\partial S_{1}}{\partial G} & =\sigma_{4} \sum_{i=1}^{3} \frac{\partial Q_{i}}{\partial G} f_{i}+\sigma_{4} \sum_{i=1}^{3} Q_{i}\left(\frac{\partial f_{i}}{\partial e}+\frac{\partial f_{i}}{\partial v} \frac{\partial v}{\partial e}\right) \frac{\partial e}{\partial G} \\
& =\sigma_{4} \sum_{i=1}^{3} \frac{\partial Q_{i}}{\partial G} f_{i}+\frac{\partial S_{1}}{\partial L} \frac{\partial e}{\partial G}\left(\frac{\partial e}{\partial L}\right)^{-1}
\end{aligned}
$$


From (6.37) through (6.39), with the same approximations used in obtaining (7.05), we find

$$
\begin{aligned}
& \frac{\partial Q_{1}}{\partial G}=\frac{3}{64}\left(\frac{r_{e}}{p}\right)^{4}\left(136-500 \eta_{0}^{2}+385 \eta_{0}^{4}\right) \\
& \frac{\partial Q_{2}}{\partial G}=-\frac{5}{16}\left(\frac{r_{e}}{p}\right)^{4}\left(12-82 \eta_{0}^{2}+77 \eta_{0}^{4}\right) \\
& \frac{\partial Q_{3}}{\partial G}=\frac{35}{64}\left(\frac{r_{e}}{p}\right)^{4} \eta_{0}^{2}\left(11 \eta_{0}^{2}-4\right) .
\end{aligned}
$$

Next we need the elliptic approximations

$$
\begin{aligned}
& \frac{\partial e}{\partial L}=(\mu a)^{-1 / 2} e^{-1}\left(1-e^{2}\right) \\
& \frac{\partial e}{\partial G}=-e^{-1}\left(\frac{1-e^{2}}{\mu a}\right)^{1 / 2} \\
& \frac{\partial v}{\partial e}=\left(\frac{1}{1-e^{2}}+\frac{a}{r}\right) \sin v=\left(1-e^{2}\right)^{-1}\left(2 \sin v+\frac{1}{2} e \sin 2 v\right)
\end{aligned}
$$

To compute the $\partial S_{1} / \partial L$ and $\partial S_{1} / \partial G$ we need finally the six derivatives $\partial f_{i} / \partial e$ and $\partial f_{i} / \partial v, i=1,2,3$. From (7.08) through (7.10) we find

$$
\begin{gathered}
\frac{\partial f_{1}}{\partial e}=3 e(v-l)+\left(3+\frac{9 e^{2}}{4}\right) \sin v+\frac{3}{2} e \sin 2 v+\frac{e^{3}}{4} \sin 3 v \\
\frac{\partial f_{1}}{\partial v}=1+\frac{3}{2} e^{2}+\left(3 e+\frac{3 e^{3}}{4}\right) \cos v+\frac{3}{2} e^{2} \cos 2 v+\frac{e^{3}}{4} \cos 3 v
\end{gathered}
$$

$\frac{\partial f_{2}}{\partial e}=\frac{3}{2} e(v-l) \cos 2 g+\frac{3 e^{2}}{8} \sin (v-2 g)+\left(\frac{3}{2}+\frac{9 e^{2}}{8}\right) \sin (v+2 g)$

$$
+\frac{3}{2} e \sin (2 v+2 g)+\left(\frac{1}{2}+\frac{3 e^{2}}{8}\right) \sin (3 v+2 g)+\frac{3 e}{8} \sin (4 v+2 g)+\frac{3 e^{2}}{40} \sin (5 v+2 g)
$$

$$
\begin{aligned}
\frac{\partial f_{2}}{\partial v}=\frac{3}{4} e^{2} \cos 2 g+\frac{e^{3}}{8} \cos (v- & 2 g)+\left(\frac{3}{2} e+\frac{3 e^{3}}{8}\right) \cos (v+2 g)+\left(1+\frac{3}{2} e^{2}\right) \cos (2 v+2 g) \\
+ & \left(\frac{3}{2} e+\frac{3 e^{3}}{8}\right) \cos (3 v+2 g)+\frac{3 e^{2}}{4} \cos (4 v+2 g)+\frac{e^{3}}{8} \cos (5 v+2 g)
\end{aligned}
$$

$\frac{\partial f_{3}}{\partial e}=\frac{3 e^{2}}{8} \sin (v+4 g)+\frac{3 e}{4} \sin (2 v+4 g)+\left(\frac{1}{2}+\frac{3 e^{2}}{8}\right) \sin (3 v+4 g)+\frac{3 e}{4} \sin (4 v+4 g)$

$$
+\left(\frac{3}{10}+\frac{9 e^{2}}{40}\right) \sin (5 v+4 g)+\frac{e}{4} \sin (6 v+4 g)+\frac{3 e^{2}}{56} \sin (7 v+4 g)
$$

$\frac{\partial f_{3}}{\partial v}=\frac{e^{3}}{8} \cos (v+4 g)+\frac{3 e^{2}}{4} \cos (2 v+4 g)+\left(\frac{3 e}{2}+\frac{3 e^{3}}{8}\right) \cos (3 v+4 g)+\left(1+\frac{3 e^{2}}{2}\right) \cos (4 v+4 g)$

$$
+\left(\frac{3 e}{2}+\frac{3 e^{3}}{8}\right) \cos (5 v+4 g)+\frac{3 e^{2}}{4} \cos (6 v+4 g)+\frac{e^{3}}{8} \cos (7 v+4 g) .
$$

There then follow

$$
\begin{aligned}
& \left(1-e^{2}\right)\left(\frac{\partial f_{1}}{\partial e}+\frac{\partial f_{1}}{\partial v} \frac{\partial v}{\partial e}\right)=3 e\left(1-e^{2}\right)(v-l)+\left(5+\frac{3 e^{2}}{2}-\frac{17 e^{4}}{8}\right) \sin v+\left(5 e-\frac{e^{3}}{4}\right) \sin 2 v \\
& +\left(\frac{5 e^{2}}{2}-\frac{e^{4}}{16}\right) \sin 3 v+\frac{5 e^{3}}{8} \sin 4 v+\frac{e^{4}}{16} \sin 5 v \\
& \left(1-e^{2}\right)\left(\frac{\partial f_{2}}{\partial e}+\frac{\partial f_{2}}{\partial v} \frac{\partial v}{\partial e}\right)=-\frac{e}{8}\left(14+5 e^{2}\right) \sin 2 g+\frac{3 e}{2}\left(1-e^{2}\right)(v-l) \cos 2 g \\
& +\left(\frac{3 e^{2}}{2}-\frac{9 e^{4}}{32}\right) \sin (v-2 g)+\frac{5 e^{3}}{16} \sin (2 v-2 g)+\frac{e^{4}}{32} \sin (3 v-2 g)
\end{aligned}
$$




$$
\begin{gathered}
+\left(\frac{1}{2}-\frac{3}{2} e^{2}-\frac{19 e^{4}}{16}\right) \sin (v+2 g)+\left(\frac{3}{2} e-\frac{3}{2} e^{3}\right) \sin (2 v+2 g)+\left(\frac{3}{2}+e^{2}-\frac{5 e^{4}}{16}\right) \sin (3 v+2 g) \\
+\left(\frac{17 e}{8}+\frac{e^{3}}{4}\right) \sin (4 v+2 g)+\left(\frac{6 e^{2}}{5}+\frac{3 e^{4}}{160}\right) \sin (5 v+2 g) \\
\left(1-e^{2}\right)\left(\frac{\partial f_{3}}{\partial e}+\frac{\partial f_{3}}{\partial v} \frac{\partial v}{\partial e}\right)=\frac{e^{3}}{32} \sin (6 v+2 g)+\frac{e^{4}}{32} \sin (7 v+2 g) \\
\quad-\left(e+\frac{11 e^{3}}{8}\right) \sin (2 v+4 g)-\left(\frac{1}{2}+\frac{5}{4} e^{2}+\frac{7 e^{4}}{16}\right) \sin (3 v+4 g)+\left(\frac{3 e}{4}-\frac{3 e^{2}}{4}\right) \sin (4 v+4 g) \\
+\left(\frac{13}{10}+\frac{21 e^{2}}{20}-\frac{13 e^{4}}{80}\right) \sin (5 v+4 g)+\left(2 e+\frac{3 e^{3}}{8}\right) \sin (6 v+4 g) \\
+\left(\frac{33 e^{2}}{28}+\frac{9 e^{4}}{224}\right) \sin (7 v+4 g)+\frac{5 e^{3}}{16} \sin (8 v+4 g)+\frac{e^{4}}{32} \sin (9 v+4 g)
\end{gathered}
$$

From (6.34), (7.14), and (7.16) it follows that

$$
Q_{i} \frac{\partial e}{\partial L}=-\frac{1}{8}\left(\frac{r_{e}}{p}\right)^{4}\left(\frac{1-e^{2}}{e}\right)^{3 / 2} q_{i} \quad(i=1,2,3)
$$

and then from (6.23), (7.11), and (7.23) through (7.26) that

$$
l-l^{\prime}=\sigma_{4} l_{41}
$$

where

$$
\begin{aligned}
l_{41}=\frac{\left(1-e^{2}\right)^{1 / 2}}{2048}\left(\frac{r_{e}}{p}\right)^{4}\left\{6 ( 8 - 4 0 \eta _ { 0 } ^ { 2 } + 3 5 \eta _ { 0 } ^ { 4 } ) \left[48\left(1-e^{2}\right)(v-l)+\frac{2}{e}\left(40+12 e^{2}-17 e^{4}\right) \sin v\right.\right. \\
\left.\quad+4\left(20-e^{2}\right) \sin 2 v+e\left(40-e^{2}\right) \sin 3 v+10 e^{2} \sin 4 v+e^{3} \sin 5 v\right] \\
\quad-4 \eta_{0}^{2}\left(7 \eta_{0}^{2}-6\right)\left[240\left(1-e^{2}\right)(v-l) \cos 2 g+5 e^{3} \sin (3 v-2 g)+50 e^{2} \sin (2 v-2 g)\right. \\
\quad+15 e\left(16-3 e^{2}\right) \sin (v-2 g)-20\left(14+5 e^{2}\right) \sin 2 g+\frac{10}{e}\left(8-24 e^{2}-19 e^{4}\right) \sin (v+2 g) \\
+240\left(1-e^{2}\right) \sin (2 v+2 g)+\frac{10}{e}\left(24+16 e^{2}-5 e^{4}\right) \sin (3 v+2 g)+20\left(17+2 e^{2}\right) \sin (4 v+2 g) \\
\left.+3 e\left(64+e^{2}\right) \sin (5 v+2 g)+50 e^{2} \sin (6 v+2 g)+5 e^{3} \sin (7 v+2 g)\right] \\
+\eta^{4}\left[35 e^{3} \sin (v-4 g)-350 e^{2} \sin 4 g-105 e\left(8+5 e^{2}\right) \sin (v+4 g)\right. \\
\quad-140\left(8+11 e^{2}\right) \sin (2 v+4 g)-\frac{70}{e}\left(8+20 e^{2}+7 e^{4}\right) \sin (3 v+4 g)+840\left(1-e^{2}\right) \sin (4 v+4 g) \\
+\frac{14}{e}\left(104+84 e^{2}-13 e^{4}\right) \sin (5 v+4 g)+140\left(16+3 e^{2}\right) \sin (6 v+4 g) \\
\left.\left.\quad+15 e\left(88+3 e^{2}\right) \sin (7 v+4 g)+350 e^{2} \sin (8 v+4 g)+35 e^{3} \sin (9 v+4 g)\right]\right\} .
\end{aligned}
$$

From (6.23), (7.12), (7.14), and (7.15), we find

$$
g-g^{\prime}=-\left(1-e^{2}\right)^{-1 / 2}\left(l-l^{\prime}\right)-\sigma_{4} \sum_{i=1}^{3} \frac{\partial Q_{i}}{\partial G_{i}} f_{i} .
$$


Then, from (7.13) and (7.08) through (7.10), we find

$$
g-g^{\prime}=\sigma_{4} g_{41},
$$

where

$$
\begin{aligned}
& g_{41}=-\left(1-e^{2}\right)^{-1 / 2} l_{41}-\frac{1}{512}\left(\frac{r_{e}}{p}\right)^{4}\left\{2 ( 1 3 6 - 5 0 0 \eta _ { 0 } ^ { 2 } + 3 8 5 \eta _ { 0 } ^ { 4 } ) \left[6\left(2+3 e^{2}\right)(v-l)\right.\right. \\
& \left.+9 e\left(4+e^{2}\right) \sin v+9 e^{2} \sin 2 v+e^{3} \sin 3 v\right]-2\left(12-82 \eta_{0}^{2}+11 \eta_{0}^{4}\right)\left[60 e^{2}(v-l) \cos 2 g\right. \\
& +10 e^{3} \sin (v-2 g)+30 e\left(4+e^{2}\right) \sin (v+2 g)+20\left(2+3 e^{2}\right) \sin (2 v+2 g) \\
& \left.+10 e\left(4+e^{2}\right) \sin (3 v+2 g)+15 e^{2} \sin (4 v+2 g)+2 e^{3} \sin (5 v+2 g)\right] \\
& +\eta_{0}^{2}\left(11 \eta_{0}^{2}-4\right)\left[35 e^{3} \sin (v+4 g)+105 e^{2} \sin (2 v+4 g)+35 e\left(4+e^{2}\right) \sin (3 v+4 g)\right. \\
& +35\left(2+3 e^{2}\right) \sin (4 v+4 g)+21 e\left(4+3 e^{2}\right) \sin (5 v+4 g) \\
& \left.\left.+35 e^{2} \sin (6 v+4 g)+5 e^{3} \sin (7 v+4 g)\right]\right\} .
\end{aligned}
$$

This concludes the solution for the short-periodic terms arising from the residual fourth harmonic, with coefficient $\sigma_{4} \equiv J_{4}+J_{2}^{2}$.

\section{Long-Periodic Terms}

By $(6.21)$ and (6.28) the Hamiltonian $F^{*}$ is

$$
F^{*}=F_{0}\left(L^{\prime}, G^{\prime}, H\right)+F_{1 c}\left(L^{\prime}, G^{\prime}, H\right)+F_{1 p}\left(L^{\prime}, G^{\prime}, H, g^{\prime}\right),
$$

short-periodic terms having been eliminated. We now try to find new canonical variables $L^{\prime \prime}$, $G^{\prime \prime}, H^{\prime \prime}, l^{\prime \prime}, g^{\prime \prime}$, and $h^{\prime \prime}$, corresponding to a new Hamiltonian $F_{0}^{* *}\left(L^{\prime \prime}, G^{\prime \prime}, H^{\prime \prime}\right)+F_{1}^{* *}\left(L^{\prime \prime}, G^{\prime \prime}, H^{\prime \prime}\right)$, so that

$$
F_{0}\left(L^{\prime}, G^{\prime}, H\right)+F_{1 c}\left(L^{\prime}, G^{\prime}, H\right)+F_{1 p}\left(L^{\prime}, G^{\prime}, H, g^{\prime}\right)=F_{0}^{* *}\left(L^{\prime \prime}, G^{\prime \prime}, H^{\prime \prime}\right)+F_{1}^{* *}\left(L^{\prime \prime}, G^{\prime \prime}, H^{\prime \prime}\right) .
$$

If we can do so, then $L^{\prime \prime}, G^{\prime \prime}$, and $H^{\prime \prime}$ will be constants of the motion and

$$
\dot{l}^{\prime \prime}=-\frac{\partial F^{* *}}{\partial L^{\prime \prime}} \quad \dot{g}^{\prime \prime}=-\frac{\partial F^{* *}}{\partial G^{\prime \prime}} \quad \dot{h}^{\prime \prime}=-\frac{\partial F^{* *}}{\partial H^{\prime \prime}} .
$$

To find the necessary canonical transformation, we introduce the generating function

$$
S^{*}=L^{\prime \prime} l^{\prime}+G^{\prime \prime} g^{\prime}+H^{\prime \prime} h^{\prime}+S_{1}^{*}\left(L^{\prime \prime}, G^{\prime \prime}, H^{\prime \prime}, g^{\prime}\right)
$$

where $S_{1}^{*}$ is to be of order $\sigma_{4}$. Then

$$
\begin{aligned}
& L^{\prime}=\frac{\partial S^{*}}{\partial l^{\prime}}=L^{\prime \prime} \\
& l^{\prime \prime}=\frac{\partial S^{*}}{\partial L^{\prime \prime}}=l^{\prime}+\frac{\partial S_{1}^{*}}{\partial L^{\prime \prime}} \\
& G^{\prime}=\frac{\partial S^{*}}{\partial g^{\prime}}=G^{\prime \prime}+\frac{\partial S_{1}^{*}}{\partial g^{\prime}} \\
& g^{\prime \prime}=\frac{\partial S^{*}}{\partial G^{\prime \prime}}=g^{\prime}+\frac{\partial S_{1}^{*}}{\partial G^{\prime \prime}} \\
& H=H^{\prime}=\frac{\partial S^{*}}{\partial h^{\prime}}=H^{\prime \prime} \\
& h^{\prime \prime}=\frac{\partial S^{*}}{\partial H^{\prime \prime}}=h^{\prime}+\frac{\overline{\partial S_{1}^{*}}}{\partial H}
\end{aligned}
$$


Insertion of (8.04) and (8.05) into (8.01) leads to

$$
\begin{aligned}
& F_{0}\left(L^{\prime}, G^{\prime \prime}+\frac{\partial S_{1}^{*}}{\partial g^{\prime}}, H\right)+F_{1 c}\left(L^{\prime}, G^{\prime \prime}+\frac{\partial S_{1}^{*}}{\partial g^{\prime}}, H\right)+F_{1 p}\left(L^{\prime}, G^{\prime \prime}+\frac{\partial S_{1}^{*}}{\partial g^{\prime}}, H, g^{\prime}+\frac{\partial S_{1}^{*}}{\partial G^{\prime \prime}}\right) \\
& =F_{0}^{* *}+F_{1}^{* *}
\end{aligned}
$$

whose Taylor expansion, with neglect of terms of order $\sigma_{4}^{2}$ or higher, is

$$
F_{0}\left(L^{\prime}, G^{\prime \prime}, H\right)+\frac{\partial F_{0}}{\partial G^{\prime \prime}} \frac{\partial S_{1}^{*}}{\partial g^{\prime}}+F_{1 c}\left(L^{\prime}, G^{\prime \prime}, H\right)+F_{1 p}\left(L^{\prime}, G^{\prime \prime}, H, g^{\prime}\right)=F_{0}^{* *}+F_{1}^{* *} .
$$

Splitting (8.07) into zeroth order and first order terms yields, respectively,

$$
\begin{gathered}
F_{0}^{* *}=F_{0}\left(L^{\prime}, G^{\prime \prime}, H\right) \\
\frac{\partial F_{0}}{\partial G^{\prime \prime}} \frac{\partial S_{1}^{*}}{\partial g^{\prime}}+F_{1 c}+F_{1 p}=F_{1}^{* *} .
\end{gathered}
$$

Resolution of (8.09) into constant and long-periodic terms then shows that

$$
\begin{gathered}
F_{1}^{* *}=F_{1 c}\left(L^{\prime}, G^{\prime \prime}, H\right) \\
\frac{\partial F_{0}}{\partial G^{\prime \prime}} \frac{\partial S_{1}^{*}}{\partial g^{\prime}}=-F_{1 p}\left(L^{\prime}, G^{\prime \prime}, H, g^{\prime}\right) .
\end{gathered}
$$

With use of (4.17) and (6.13) and of double primes to denote quantities corresponding to $G^{\prime \prime},(8.11)$ becomes

$$
2 \pi\left(\nu_{1}^{\prime \prime}-\nu_{2}^{\prime \prime}\right) \frac{\partial S_{1}^{*}}{\partial g^{\prime}}=\frac{15 \mu r_{e}^{4} \sigma_{4}}{32 a^{\prime 5}} e^{\prime \prime 2}\left(1-e^{\prime \prime 2}\right)^{-7 / 2} \eta_{0}^{\prime \prime 2}\left(3-\frac{7}{2} \eta_{0}^{\prime \prime 2}\right) \cos 2 g^{\prime}
$$

By eqs (7.34) and (7.37) of an earlier paper [Vinti 1961 a], we have

$$
\begin{aligned}
2 \pi\left(\nu_{1}^{\prime \prime}-\nu_{2}^{\prime \prime}\right) & =-\frac{3 r_{e}^{2} J_{2} n}{4 p^{2}}\left(5 \cos ^{2} I-1\right)+\mathrm{O}\left(J_{2}^{2}\right) \\
& =\frac{3}{4} \frac{\mu^{4} r_{e}^{2} J_{2}}{L^{\prime} G^{\prime \prime}}\left(1-\frac{5 H^{2}}{G^{\prime 2}}\right)+\mathrm{O}\left(J_{2}^{2}\right) .
\end{aligned}
$$

On inserting (8.13) into (8.12) and replacing elements by Delaunay variables, we find

$$
\frac{\partial S_{1}^{*}}{\partial g^{\prime}}=-\frac{5 \mu^{2} r_{e}^{2} \sigma_{4}}{16 J_{2} G^{\prime \prime 3}}\left(1-\frac{G^{\prime 2}}{L^{\prime 2}}\right)\left(1-\frac{H^{2}}{G^{\prime \prime 2}}\right)\left(1-7 \frac{H^{2}}{G^{\prime \prime 2}}\right)\left(1-5 \frac{H^{2}}{G^{\prime 2}}\right)^{-1} \cos 2 g^{\prime} .
$$

Integration then yields

$$
S_{1}^{*}=-\frac{5 \mu^{2} r_{e}^{2} \sigma_{4}}{32 J_{2} G^{\prime \prime 3}}\left(1-\frac{G^{\prime \prime 2}}{L^{\prime 2}}\right)\left(1-\frac{H^{2}}{G^{\prime \prime 2}}\right)\left(1-7 \frac{H^{2}}{G^{\prime \prime 2}}\right)\left(1-5 \frac{H^{2}}{G^{\prime 2}}\right)^{-1} \sin 2 g^{\prime} .
$$

Then

$$
\begin{aligned}
& \frac{\partial S_{1}^{*}}{\partial L^{\prime}}=-\frac{5 \mu^{2} r_{e}^{2} \sigma_{4}}{16 J_{2} G^{\prime \prime} L^{\prime 3}}\left(1-\frac{H^{2}}{G^{\prime \prime 2}}\right)\left(1-7 \frac{H^{2}}{G^{\prime \prime 2}}\right)\left(1-5 \frac{H^{2}}{G^{\prime \prime 2}}\right)^{-1} \sin 2 g^{\prime} \\
& \frac{\partial S_{1}^{*}}{\partial G^{\prime \prime}}=\frac{5 \mu^{2} r_{e}^{2} \sigma_{4}}{32 J_{2} G^{\prime \prime 4}}\left[3\left(1-5 \frac{H^{2}}{G^{\prime \prime 2}}\right)-\frac{G^{\prime \prime 2}}{L^{\prime 2}}\left(1-9 \frac{H^{2}}{G^{\prime \prime 2}}\right)-\frac{8 H^{4}}{G^{\prime \prime 4}}\left(7-5 \frac{G^{\prime \prime 2}}{L^{\prime 2}}\right)\left(1-5 \frac{H^{2}}{G^{\prime \prime 2}}\right)^{-1}\right. \\
& \left.-80 \frac{H^{6}}{G^{\prime \prime 6}}\left(1-\frac{G^{\prime \prime 2}}{L^{\prime 2}}\right)\left(1-5 \frac{H^{2}}{G^{\prime \prime 2}}\right)^{-2}\right] \sin 2 g^{\prime}
\end{aligned}
$$




$$
\frac{\partial S_{1}^{*}}{\partial H}=\frac{5 \mu^{2} r_{e}^{2} \sigma_{4}}{16 J_{2}} \frac{H}{G^{\prime \prime 5}}\left(1-\frac{G^{\prime \prime 2}}{L^{\prime 2}}\right)\left[3+16 \frac{H^{2}}{G^{\prime \prime 2}}\left(1-5 \frac{H^{2}}{G^{\prime 2}}\right)^{-1}+40 \frac{H^{4}}{G^{\prime \prime 4}}\left(1-5 \frac{H^{2}}{G^{\prime \prime 2}}\right)^{-2}\right] \sin 2 g^{\prime} .
$$

We now express the long-periodic terms in the notation of (5.00) and (5.01).

$$
\begin{aligned}
G^{\prime}-G^{\prime \prime} & =\frac{\sigma_{4}}{J_{2}} \tilde{G}_{4} & g^{\prime}-g^{\prime \prime} & =\frac{\sigma_{4}}{J_{2}} \tilde{g}_{4} \\
l^{\prime}-l^{\prime \prime} & =\frac{\sigma_{4}}{J_{2}} \tilde{l}_{4} & h^{\prime}-h^{\prime \prime} & =\frac{\sigma_{4}}{J_{2}} \tilde{h}_{4} .
\end{aligned}
$$

If we then use (8.04), (8.05), (8.14), and (8.16) through (8.19) and place $G^{\prime 2}=\mu p, L^{\prime 2}=\mu a, H / G^{\prime \prime}$ $=\cos I, G^{\prime \prime 2} / L^{\prime 2}=1-e^{2}, g^{\prime}=$ unperturbed $g$ as given by (4.18), we find to the required accuracy

$$
\begin{aligned}
& \tilde{G}_{4}=-\frac{5 r_{e}^{2} n}{16} e^{2}\left(1-e^{2}\right)^{-3 / 2} \eta_{0}^{2}\left(1-7 \cos ^{2} I\right)\left(1-5 \cos ^{2} I\right)^{-1} \cos 2 g \\
& \tilde{l}_{4}=\frac{5}{16}\left(\frac{r_{e}}{a}\right)^{2}\left(1-e^{2}\right)^{-1 / 2} \eta_{0}^{2}\left(1-7 \cos ^{2} I\right)\left(1-5 \cos ^{2} I\right)^{-1} \sin 2 g \\
& \tilde{g}_{4}=-\frac{5}{32}\left(\frac{r_{e}}{p}\right)^{2}\left[2+e^{2}-3\left(2+3 e^{2}\right) \cos ^{2} I-8\left(2+5 e^{2}\right) \cos ^{4} I\left(1-5 \cos ^{2} I\right)^{-1}\right. \\
& \left.\quad-80 e^{2} \cos ^{6} I\left(1-5 \cos ^{2} I\right)^{-2}\right] \sin 2 g \\
& \tilde{h}_{4}=-\frac{5}{16}\left(\frac{r_{e}}{p}\right)^{2} e^{2} \cos I\left[3+16 \cos ^{2} I\left(1-5 \cos ^{2} I\right)^{-1}+40 \cos ^{4} I\left(1-5 \cos ^{2} I\right)^{-2}\right] \sin 2 g .
\end{aligned}
$$

\section{Secular Effects}

We now have to use (8.02), (8.08), (8.10), and (4.17) to obtain $l^{\prime \prime}, g^{\prime \prime}$, and $h^{\prime \prime}$ as linear functions of the time. From (4.17) and (8.08) we obtain

$$
\frac{\partial F_{0}^{* *}}{\partial L^{\prime}}=-2 \pi \nu_{1}^{\prime \prime} \quad \frac{\partial F_{0}^{* *}}{\partial G^{\prime \prime}}=2 \pi\left(\nu_{1}^{\prime \prime}-\nu_{2}^{\prime \prime}\right) \quad \frac{\partial F_{0}^{* *}}{\partial H}=2 \pi\left(\nu_{2}^{\prime \prime} \operatorname{sgn} \alpha_{3}-\nu_{3}^{\prime \prime}\right) .
$$

From (8.10) and (6.12), with use of $e^{\prime 2}=1-G^{\prime \prime 2} / L^{\prime 2}, L^{\prime 2}=\mu a^{\prime}$, and $\eta_{0}^{\prime \prime 2}=1-H^{2} / G^{\prime \prime 2}$, we have with sufficient accuracy

$$
F_{1}^{* *}=-\frac{3 \mu^{6} r_{e}^{4} \sigma_{4}}{128 L^{\prime 3} G^{\prime \prime 7}}\left(5-3 \frac{G^{\prime \prime 2}}{L^{\prime 2}}\right)\left(3-30 \frac{H^{2}}{G^{\prime 2}}+35 \frac{H^{4}}{G^{\prime \prime 4}}\right)
$$

Then

$$
\begin{aligned}
& \frac{\partial F_{1}^{* *}}{\partial L^{\prime}}=\frac{45 \mu^{6} r_{e}^{4} \sigma_{4}}{128 L^{\prime 4} G^{\prime \prime 7}}\left(1-\frac{G^{\prime \prime 2}}{L^{\prime 2}}\right)\left(3-30 \frac{H^{2}}{G^{\prime \prime 2}}+35 \frac{H^{4}}{G^{\prime \prime 4}}\right) \\
& \frac{\partial F_{1}^{* *}}{\partial G^{\prime \prime}}=\frac{15 \mu^{6} r_{e}^{4} \sigma_{4}}{128 L^{\prime 3} G^{\prime \prime 8}}\left[3\left(7-3 \frac{G^{\prime \prime 2}}{L^{\prime 2}}\right)+18\left(7 \frac{G^{\prime 2}}{L^{\prime 2}}-15\right) \frac{H^{2}}{G^{\prime \prime 2}}+7\left(55-27 \frac{G^{\prime \prime 2}}{L^{\prime 2}}\right) \frac{H^{4}}{G^{\prime 4}}\right] \\
& \frac{\partial F_{1}^{* *}}{\partial H}=\frac{15 \mu^{6} r_{e}^{4} \sigma_{4}}{32 L^{\prime 3} G^{\prime \prime 9}} H\left(5-3 \frac{G^{\prime \prime 2}}{L^{\prime 2}}\right)\left(3-7 \frac{H^{2}}{G^{\prime \prime 2}}\right) .
\end{aligned}
$$

Since our constant orbital elements $a, e$, and $I$, and thus the frequencies $\nu_{1}$ and $\nu_{2}$, correspond to $L^{\prime}, G^{\prime \prime}$, and $H$, we may drop the double primes from the $\nu^{\prime}$ s in (9.00). Also, to our specified accuracy, we may put $e^{2}=1-G^{\prime \prime 2} / L^{\prime 2}, L^{\prime 2}=\mu a$, and $\eta_{0}^{2}=1-H^{2} / G^{\prime \prime 2}$ in (9.02) through (9.04). Then, 
with the unperturbed values $l, g$, and $h$ given by (4.18), we find

$$
l^{\prime \prime}=l+\sigma_{4} l_{42} \quad g^{\prime \prime}=g+\sigma_{4} g_{42} \quad h^{\prime \prime}=h+\sigma_{4} h_{42},
$$

where

$$
\begin{gathered}
l_{42}=-\frac{45}{128}\left(\frac{r_{e}}{p}\right)^{4} n\left(1-e^{2}\right)^{1 / 2}\left(3-30 \cos ^{2} I+35 \cos ^{4} I\right) t \\
g_{42}=-\frac{15}{128}\left(\frac{r_{e}}{p}\right)^{4} n\left[3\left(4+3 e^{2}\right)-18\left(8+7 e^{2}\right) \cos ^{2} I+7\left(28+27 e^{2}\right) \cos ^{4} I\right] t \\
h_{42}=-\frac{15}{32}\left(\frac{r_{e}}{p}\right)^{4} n\left(2+3 e^{2}\right) \cos I\left(3-7 \cos ^{2} I\right) t .
\end{gathered}
$$

We postpone summarizing the results for the residual fourth harmonic until the complete algorithm in section 11 .

\section{Effects of the Third Zonal Harmonic}

By (2.01) the contribution of the third zonal harmonic to the potential is

$$
\Delta V=\mu r_{e}^{3} r^{-4} J_{3} P_{3}(\sin \theta)
$$

Corresponding to (3.02), this leads to

$$
\Delta F_{1}=-\mu r_{e}^{3} r^{-4} J_{3} P_{3}(\sin \theta)
$$

and thus to

$$
\Delta F_{1}=-\frac{\mu r_{e}^{3}}{a^{4}} J_{3}\left[-\frac{3}{2} \eta_{0}+\frac{15}{8} \eta_{0}^{3}\left(\frac{a}{r}\right)^{4} \sin (v+g)-\frac{5}{8} \eta_{0}^{3}\left(\frac{a}{r}\right)^{4} \sin (3 v+3 g)\right],
$$

corresponding to (6.06). Following the notation of section 6 , we then find

$$
\Delta F_{1 m}=(2 \pi)^{-1} \int_{0}^{2 \pi} \Delta F_{1} d l=-\frac{\mu r_{e}^{3}}{a^{4}} J_{3} e\left(1-e^{2}\right)^{-5 / 2}\left(-\frac{3}{2} \eta_{0}+\frac{15}{8} \eta_{0}^{3}\right) \sin g,
$$

which is purely long-periodic, so that

$$
\Delta F_{1 m}=\Delta F_{1 p} \quad(10.04) \quad \Delta F_{1 c}=0 .
$$

Since $\Delta F_{1 c}=0$, there are no secular effects.

The short-periodic part of $\Delta F_{1}$ is then

$$
\begin{aligned}
\Delta F_{1 l}=\Delta F_{1}-\Delta F_{1 m}=-\frac{\mu r_{e}^{3}}{a^{4}} J_{3}\left[( - \frac { 3 } { 2 } \eta _ { 0 } + \frac { 1 5 } { 8 } \eta _ { 0 } ^ { 3 } ) \left\{\left(\frac{a}{r}\right)^{4} \sin (v+g)\right.\right. & \\
& \left.\left.-e\left(1-e^{2}\right)^{-5 / 2} \sin g\right\}-\frac{5}{8} \eta_{0}^{3}\left(\frac{a}{r}\right)^{4} \sin (3 v+3 g)\right]
\end{aligned}
$$

On following the same procedure as in section 6 , we find

$$
\frac{\partial \Delta S_{1}}{\partial l}=-\left(2 \pi \nu_{1}\right)^{-1} \Delta F_{1 l}
$$


where

$$
\begin{gathered}
\Delta S_{1}=-\left(2 \pi \nu_{1}\right)^{-1} \int \Delta F_{1 l} d l \\
=-\frac{\mu r_{e}^{3}}{96 n a^{4}} J_{3}\left(1-e^{2}\right)^{-5 / 2} \eta_{0}\left\{3 ( 1 - 5 \operatorname { c o s } ^ { 2 } I ) \left[12 e(v-l) \sin g+3 e^{2} \cos (v-g)\right.\right. \\
\left.-6\left(2+e^{2}\right) \cos (v+g)-6 e \cos (2 v+g)-e^{2} \cos (3 v+g)\right]+\eta_{0}^{2}\left[15 e^{2} \cos (v+3 g)+30 e \cos (2 v+3 g)\right. \\
\left.\left.+10\left(2+e^{2}\right) \cos (3 v+3 g)+15 e \cos (4 v+3 g)+3 e^{2} \cos (5 v+3 g)\right]\right\}
\end{gathered}
$$

Before taking derivatives of (10.09) with respect to $L^{\prime}$ and $G^{\prime}$ one must replace $e^{2}$ by $1-G^{\prime 2} / L^{\prime 2}, \eta_{0}^{2}$ by $1-H^{2} / G^{\prime 2}$, and $a$ by $L^{\prime 2} / \mu$. The short-periodic effects are then given by

$$
\begin{array}{lll}
\frac{\partial \Delta S_{1}}{\partial l}=L-L^{\prime}=J_{3} L_{3} & -\frac{\partial \Delta S_{1}}{\partial L^{\prime}}=l-l^{\prime}=J_{3} l_{3} \\
\frac{\partial \Delta S_{1}}{\partial g}=G-G^{\prime}=J_{3} G_{3} & (10.10) & -\frac{\partial \Delta S_{1}}{\partial G^{\prime}}=g-g^{\prime}=J_{3} g_{3} \\
\frac{\partial \Delta S_{1}}{\partial h}=H-H^{\prime}=0 & -\frac{\partial \Delta S_{1}}{\partial H}=h-h^{\prime}=J_{3} h_{3},
\end{array}
$$

where

$$
\begin{aligned}
& L_{3}=-\frac{n r_{e}^{3}}{a}\left\{\left(-\frac{3}{2} \eta_{0}+\frac{15}{8} \eta_{0}^{3}\right)\left[\left(\frac{a}{r}\right)^{4} \sin (v+g)-e\left(1-e^{2}\right)^{-5 / 2} \sin g\right]\right. \\
& \left.\left.-\frac{5}{8} \eta_{0}^{3}\left(\frac{a}{r}\right)^{4} \sin (3 v+3 g)\right]\right\} \\
& G_{3}=-n a^{3}\left(1-e^{2}\right)^{1 / 2}\left(\frac{r_{e}}{p}\right)^{3}\left\{( - \frac { 3 } { 2 } \eta _ { 0 } + \frac { 1 5 } { 8 } \eta _ { 0 } ^ { 3 } ) \left[e(v-l) \cos g+\frac{e^{2}}{4} \sin (v-g)\right.\right. \\
& \left.+\left(1+\frac{1}{2} e^{2}\right) \sin (v+g)+\frac{1}{2} e \sin (2 v+g)+\frac{e^{2}}{12} \sin (3 v+g)\right]-\frac{15}{8} \eta_{0}^{3}\left[\frac{e^{2}}{4} \sin (v+3 g)+\frac{1}{2} e \sin (2 v+3 g)\right. \\
& \left.\left.+\frac{1}{3}\left(1+\frac{1}{2} e^{2}\right) \sin (3 v+3 g)+\frac{e}{4} \sin (4 v+3 g)+\frac{e^{2}}{20} \sin (5 v+3 g)\right]\right\} \text {. } \\
& l_{3}=\frac{\eta_{0}}{8 e}\left(1-e^{2}\right)^{1 / 2}\left(\frac{r_{e}}{p}\right)^{3}\left\{3 ( 5 \eta _ { 0 } ^ { 2 } - 4 ) \left[\left(1-e^{2}\right)(v-l) \sin g+\left(1+\frac{1}{2} e^{2}\right) \cos g\right.\right. \\
& \dashv-\frac{e}{8}\left(14-3 e^{2}\right) \cos (v-g)+\frac{1}{2} e^{2} \cos (2 v-g)+\frac{e^{3}}{16} \cos (3 v-g)-e\left(1-e^{2}\right) \cos (v+g) \\
& \left.-\frac{3}{2} \cos (2 v+g)-\frac{e}{24}\left(34-e^{2}\right) \cos (3 v+g)-\frac{1}{2} e^{2} \cos (4 v+g)-\frac{e^{3}}{16} \cos (5 v+g)\right] \\
& +5 \eta_{0}^{2}\left[-\frac{1}{2} e^{2} \cos 3 g-\frac{e^{3}}{16} \cos (v-3 g)-\frac{e}{8}\left(6+5 e^{2}\right) \cos (v+3 g)-\left(\frac{1}{2}+e^{2}\right) \cos (2 v+3 g)\right. \\
& +\frac{e}{3}\left(1-e^{2}\right) \cos (3 v+3 g)+\frac{1}{4}\left(5+e^{2}\right) \cos (4 v+3 g)+\frac{e}{40}\left(54+e^{2}\right) \cos (5 v+3 g) \\
& \left.\left.+\frac{1}{2} e^{2} \cos (6 v+3 g)+\frac{e^{3}}{16} \cos (7 v+3 g)\right]\right\} \text {. } \\
& g_{3}=-\left(1-e^{2}\right)^{-1 / 2} l_{3}-\frac{1}{8}\left(\frac{r_{e}}{p}\right)^{3}\left\{\eta _ { 0 } ^ { - 1 } ( 4 - 3 9 \eta _ { 0 } ^ { 2 } + 4 0 \eta _ { 0 } ^ { 4 } ) \left[e(v-l) \sin g+\frac{e^{4}}{4} \cos (v-g)\right.\right. \\
& \left.-\left(1+\frac{1}{2} e^{2}\right) \cos (v+g)-\frac{1}{2} e \cos (2 v+g)-\frac{e^{2}}{12} \cos (3 v+g)\right]+5 \eta_{0}\left(8 \eta_{0}^{2}-3\right)\left[\frac{e^{2}}{4} \cos (v+3 g)\right.
\end{aligned}
$$




$$
\begin{gathered}
\left.\left.+\frac{1}{2} e \cos (2 v+3 g)+\frac{1}{3}\left(1+\frac{1}{2} e^{2}\right) \cos (3 v+3 g)+\frac{e}{4} \cos (4 v+3 g)+\frac{e^{2}}{20} \cos (5 v+3 g)\right]\right\} \\
h_{3}=\left(\frac{r_{e}}{p}\right)^{3}\left\{\frac { 3 } { 2 } \operatorname { c o t } I ( 1 - \frac { 1 5 } { 4 } \operatorname { s i n } ^ { 2 } I ) \left[e(v-l) \sin g+\frac{e^{2}}{4} \cos (v-g)-\left(1+\frac{1}{2} e^{2}\right) \cos (v+g)\right.\right. \\
\left.-\frac{1}{2} e \cos (2 v+2 g)-\frac{e^{2}}{12} \cos (3 v+2 g)\right]-\frac{15}{16} \sin 2 I\left[\frac{e^{2}}{4} \cos (v+3 g)\right. \\
\left.\left.+\frac{1}{2} e \cos (2 v+3 g)+\frac{1}{3}\left(1+\frac{1}{2} e^{2}\right) \cos (3 v+3 g)+\frac{e}{4} \cos (4 v+3 g)+\frac{e^{2}}{20} \cos (5 v+3 g)\right]\right\} .
\end{gathered}
$$

Continuing on to the long-periodic effects and following the procedures of section 8 , we find

$$
\left(\Delta F_{1}\right)^{* *}=0
$$

so that the third harmonic gives no secular changes, and

$$
2 \pi\left(\nu_{1}^{\prime \prime}-\nu_{2}^{\prime \prime}\right) \frac{\partial \Delta S_{1}^{*}}{\partial g^{\prime}}=-\Delta F_{1 p}
$$

Now

$$
F_{1 p}=-\frac{3}{8} \frac{\mu^{5} r_{e}^{3} e^{\prime \prime}}{L^{\prime 3} G^{\prime \prime 5}} J_{3} \eta_{0}^{\prime \prime}\left(1-5 \frac{H^{2}}{G^{\prime \prime 2}}\right) \sin g^{\prime}
$$

by (10.03) and (10.04). With use of (10.18) and (10.19) and of (8.13) for $2 \pi\left(\nu_{1}^{\prime \prime}-\nu_{2}^{\prime \prime}\right)$, we find

$$
\frac{\partial \Delta S_{1}^{*}}{\partial g^{\prime}}=\frac{1}{2} \frac{\mu r_{e}}{G^{\prime \prime}} \frac{J_{3}}{J_{2}} e^{\prime \prime} \eta_{0}^{\prime \prime} \sin g^{\prime},
$$

so that

$$
\Delta S_{1}^{*}=-\frac{1}{2} \frac{\mu r_{e}}{G^{\prime \prime}} \frac{J_{3}}{J_{2}} e^{\prime \prime} \eta_{0}^{\prime \prime} \cos g^{\prime}
$$

It follows that

$$
\begin{aligned}
& \frac{\partial \Delta S_{1}^{*}}{\partial l^{\prime}}=L^{\prime}-L^{\prime \prime}=0 \\
& \frac{\partial \Delta S_{1}^{*}}{\partial g^{\prime}}=G^{\prime}-G^{\prime \prime}=J_{3} \tilde{G}_{3} / J_{2} \\
& \frac{\partial \Delta S_{1}^{*}}{\partial h^{\prime}}=H^{\prime}-H^{\prime \prime}=0
\end{aligned}
$$

$$
\begin{aligned}
& -\frac{\partial \Delta S_{1}^{*}}{\partial L^{\prime}}=l^{\prime}-l^{\prime \prime}=J_{3} \tilde{l}_{3} / J_{2} \\
& -\frac{\partial \Delta S_{1}^{*}}{\partial G^{\prime}}=g^{\prime}-g^{\prime \prime}=J_{3} \tilde{g}_{3} / J_{2} \\
& -\frac{\partial \Delta S_{1}^{*}}{\partial H^{\prime}}=h^{\prime}-h^{\prime \prime}=J_{3} \tilde{h}_{3} / J_{2},
\end{aligned}
$$

where

$$
\begin{gathered}
\tilde{G}_{3}=\frac{1}{2} r_{e} n a\left(1-e^{2}\right)^{-1 / 2} e \eta_{0} \sin g \\
\tilde{l}_{3}=\frac{1}{2} \frac{r_{e}}{a} \frac{\left(1-e^{2}\right)^{1 / 2}}{e} \sin I \cos g \\
\tilde{g}_{3}=\frac{1}{2} \frac{r_{e}}{p}\left(\frac{e \cos ^{2} I}{\sin I}-\frac{\sin I}{e}\right) \cos g \\
\tilde{h}_{3}=-\frac{1}{2} \frac{r_{e}}{p} e \cot I \cos g .
\end{gathered}
$$


A summary of the results for the third harmonic will appear in the next section, which gives the complete algorithm.

\section{The Complete Algorithm}

We shall summarize results by writing out the complete algorithm for the calculation of the motion in the potential field (1.00), as modified by the third harmonic and the residual fourth harmonic. This will involve a repetition, with some changes, of about two pages of an earlier paper [Vinti $1961 \mathrm{a}$ ], but it is highly desirable to assemble the whole solution in one place.

Let the planetary constants $\mu, r_{e}, J_{2}, J_{3}$, and $J_{4}$ be given, along with the constant orbital elements $a, e, I, l_{0}, g_{0}$, and $\beta_{3}$. To calculate the

\section{Unperturbed Reference Orbit}

compute

$$
\begin{aligned}
& c=r_{e} J_{2}^{1 / 2} \quad \eta_{0}=\sin I \\
& D=\left(a p-c^{2}\right)\left(a p-c^{2} \eta_{0}^{2}\right)+4 a^{2} c^{2} \eta_{0}^{2} \\
& p=a\left(1-e^{2}\right) \\
& A=-2 a c^{2} D^{-1}\left(1-\eta_{0}^{2}\right)\left(a p-c^{2} \eta_{0}^{2}\right) \\
& D^{\prime}=4 a^{2} c^{2}\left(1-\eta_{0}^{2}\right)+D \\
& b_{1}=-\frac{1}{2} A \quad b_{2}=B^{1 / 2} \\
& B=c^{2} \eta_{0}^{2} D^{-1} D^{\prime} \\
& -\frac{\alpha_{2}^{2}}{2 \alpha_{1}}=a_{0} p_{0}=-c^{2}\left(1-\eta_{0}^{2}\right)+a p D^{-1} D^{\prime} \\
& -2 \alpha_{1}=\mu\left(a+b_{1}\right)^{-1} \\
& \alpha_{3}=\alpha_{2}\left(1-\frac{c^{2} \eta_{0}^{2}}{a_{0} p_{0}}\right)^{1 / 2} \cos I \quad \eta_{2}^{-2}=\frac{c^{2} D}{a p D^{\prime}} \\
& \alpha_{2}=\left(-2 \alpha_{1}\right)^{1 / 2}\left(a_{0} p_{o}\right)^{1 / 2}>0 \\
& A_{1}=\left(1-e^{2}\right)^{1 / 2} p \sum_{n=2}^{\infty}\left(b_{2} / p\right)^{n} P_{n}\left(b_{1} / b_{2}\right) R_{n-2}\left[\left(1-e^{2}\right)^{1 / 2}\right] \\
& A_{2}=\left(1-e^{2}\right)^{1 / 2} p^{-1} \sum_{n=0}^{\infty}\left(b_{2} / p\right)^{n} P_{n}\left(b_{1} / b_{2}\right) R_{n}\left[\left(1-e^{2}\right)^{1 / 2}\right],
\end{aligned}
$$

where $P_{n}(x)$ is the Legendre polynomial of degree $n$ and where $R_{n}(x) \equiv x^{n} P_{n}\left(x^{-1}\right)$, always a polynomial of degree $[n / 2]$ in $x^{2}$.

where

$$
A_{3}=\left(1-e^{2}\right)^{1 / 2} p^{-3} \sum_{m=0}^{\infty} D_{m} R_{m+2}\left[\left(1-e^{2}\right)^{1 / 2}\right]
$$

$$
\begin{aligned}
D_{2 i} & =\sum_{n=0}^{i}(-1)^{i-n}(c / p)^{2 i-2 n}\left(b_{2} / p\right)^{2 n} P_{2 n}\left(b_{1} / b_{2}\right) \\
D_{2 i+1} & =\sum_{n=0}^{i}(-1)^{i-n}(c / p)^{2 i-2 n}\left(b_{2} / p\right)^{2 n+1} P_{2 n+1}\left(b_{1} / b_{2}\right) \\
B_{1} & =2 \pi^{-1} q^{-2}[K(q)-E(q)] \quad B_{2}=2 \pi^{-1} K(q),
\end{aligned}
$$

where $K(q)$ and $E(q)$ are the complete elliptic integrals of the first and second kinds, respectively. It may be convenient to have the series

$$
B_{1}=\frac{1}{2}+\frac{3}{16} q^{2}+\frac{15}{128} q^{4}+\ldots \quad B_{2}=1+\frac{1}{4} q^{2}+\frac{9}{64} q^{4}+\ldots
$$


Continue with

$$
\begin{aligned}
B_{3} & =1-\left(1-\eta_{2}^{-2}\right)^{-1 / 2}-\sum_{m=2}^{\infty} \gamma_{m} \eta_{2}^{-2 m}, \text { where } \gamma_{m}=\frac{(2 m) !}{2^{2 m}(m !)^{2}} \sum_{n=1}^{m-1} \frac{(2 n) ! \eta_{0}^{2 n}}{2^{2 n}(n !)^{2}} \\
A_{11} & =\frac{3}{4}\left(1-e^{2}\right)^{1 / 2} p^{-3} e\left(-2 b_{1} b_{2}^{2} p+b_{2}^{4}\right) \quad A_{12}=\frac{3}{32}\left(1-e^{2}\right)^{1 / 2} p^{-3} b_{2}^{4} e^{2} \\
A_{21} & =\left(1-e^{2}\right)^{1 / 2} p^{-1} e\left[b_{1} p^{-1}+\left(3 b_{1}^{2}-b_{2}^{2}\right) p^{-2}-\frac{9}{2} b_{1} b_{2}^{2}\left(1+\frac{e^{2}}{4}\right) p^{-3}+\frac{3}{8} b_{2}^{4}\left(4+3 e^{2}\right) p^{-4}\right] \\
A_{22} & =\left(1-e^{2}\right)^{1 / 2} p^{-1}\left[\frac{e^{2}}{8}\left(3 b_{1}^{2}-b_{2}^{2}\right) p^{-2}-\frac{9}{8} e^{2} b_{1} b_{2}^{2} p^{-3}+\frac{3}{32} b_{2}^{4}\left(6 e^{2}+e^{4}\right) p^{-4}\right] \\
A_{23} & =\left(1-e^{2}\right)^{1 / 2} p^{-1} \frac{e^{3}}{8}\left(-b_{1} b_{2}^{2} p^{-3}+b_{2}^{4} p^{-4}\right) \\
A_{24} & =\frac{3}{256}\left(1-e^{2}\right)^{1 / 2} p^{-5} b_{2}^{4} e^{4} \\
A_{31} & =\left(1-e^{2}\right)^{1 / 2} p^{-3} e\left[2+b_{1} p^{-1}\left(3+\frac{3 e^{2}}{4}\right)-p^{-2}\left(\frac{1}{2} b_{2}^{2}+c^{2}\right)\left(4+3 e^{2}\right)\right] \\
A_{32} & =\left(1-e^{2}\right)^{1 / 2} p^{-3}\left[\frac{e^{2}}{4}+\frac{3}{4} b_{1} p^{-1} e^{2}-p^{-2}\left(\frac{e^{2}}{4}+\frac{3}{2} e^{2}\right)\left(\frac{1}{2} b_{2}^{2}+c^{2}\right)\right] \\
A_{33} & =\left(1-e^{2}\right)^{1 / 2} p^{-3} e^{3}\left[\frac{b_{1}}{12} p^{-1}-\frac{1}{3} p^{-2}\left(\frac{1}{2} b_{2}^{2}+c^{2}\right)\right] \\
A_{34} & =-\frac{1}{32}\left(1-e^{2}\right)^{1 / 2} p^{-5} e^{4}\left(\frac{1}{2} b_{2}^{2}+c^{2}\right) \\
2 \pi \nu_{1} & =\left(-2 \alpha_{1}\right)^{1 / 2}\left(a+b_{1}+A_{1}+c^{2} \eta_{0}^{2} A_{2} B_{1} B_{2}^{-1}\right)^{-1} \\
2 \pi \nu_{2} & =\left(\alpha_{2}^{2}-\alpha_{3}^{2}\right)^{1 / 2} \eta_{0}^{-1} A_{2} B_{2}^{-1}\left(a+b_{1}+A_{1}+c^{2} \eta_{0}^{2} A_{2} B_{1} B_{2}^{-1}\right)^{-1} .
\end{aligned}
$$

The uniformising variables $E, v$, and $\psi$ are then given by $E=M_{s}+E_{p}, v=M_{s}+E_{p}$, and $\psi=\psi_{s}$ $+\psi_{p}$. If $t$ is the time, their secular parts $M_{s}$ and $\psi_{s}$ are given exactly by

$$
M_{s}=l_{0}+2 \pi \nu_{1} t \quad \psi_{s}=l_{0}+g_{0}+2 \pi \nu_{2} t .
$$

Let the periodic parts be split as follows: $E_{p}=E_{0}+E_{1}+E_{2}, v_{p}=v_{0}+v_{1}+v_{2}$, and $\psi_{p}=\psi_{0}+\psi_{1}$ $+\psi_{2}$, where, e.g., $E_{0}$ contains terms of order $J_{2}^{0}, J_{2}$, and $J_{2}^{2}, E_{1}$ contains terms of order $J_{2}$ and $J_{2}^{2}$, and $E_{2}$ contains terms of order $J_{2}^{2}$ only.

Then $E_{0}$ is given by the Kepler equation

$$
M_{s}+E_{0}-e^{\prime} \sin \left(M_{s}+E_{0}\right)=M_{s},
$$

where $e^{\prime} \equiv a e\left(a+b_{1}\right)^{-1}<e$. The term $v_{0}$ is then given by placing $v=M_{s}+v_{0}$ and $E=M_{s}+v_{0}$ in the anomaly connections

$$
\cos v=(\cos E-e)(1-e \cos E)^{-1} \quad \sin v=\left(1-e^{2}\right)^{1 / 2}(1-e \cos E)^{-1} \sin E
$$

or equivalent relations. (Note that $e$ here is the original $e$ and not the $e^{\prime}$ in the Kepler equation.) Then

$$
\psi_{0}=\left(-2 \alpha_{1}\right)^{-1 / 2}\left(\alpha_{2}^{2}-\alpha_{3}^{2}\right)^{1 / 2} \eta_{0}^{-1} A_{2} B_{2}^{-1} v_{0} .
$$

The term $E_{1}$ is now given by

$$
E_{1}=\left[1-e^{\prime} \cos \left(M_{s}+E_{0}\right)\right]^{-1} M_{1}-\frac{1}{2} e^{\prime}\left[1-e^{\prime} \cos \left(M_{s}+E_{0}\right)\right]^{-3} M_{1}^{2} \sin \left(M_{s}+E_{0}\right),
$$


where

$$
M_{1}=\left(a+b_{1}\right)^{-1}\left[-\left(A_{1}+c^{2} \eta_{0}^{2} A_{2} B_{1} B_{2}^{-1}\right) v_{0}+\frac{c^{2}}{4}\left(-2 \alpha_{1}\right)^{1 / 2}\left(\alpha_{2}^{2}-\alpha_{3}^{2}\right)^{-1 / 2} \eta_{0}^{3} \sin \left(2 \psi_{s}+2 \psi_{0}\right)\right] .
$$

The term $v_{1}$ is then given by placing $v=M_{s}+v_{0}+v_{1}$ and $E=M_{s}+E_{0}+E_{1}$ in the anomaly connections. Then

$$
\begin{aligned}
\psi_{1}=\left(-2 \alpha_{1}\right)^{-1 / 2}\left(\alpha_{2}^{2}-\alpha_{3}^{2}\right)^{1 / 2} \eta_{0}^{-1} B_{2}^{-1}\left[A_{2} v_{1}+A_{21} \sin \left(M_{s}+v_{0}\right)+A_{22} \sin \left(2 M_{s}+2 v_{0}\right)\right] & \\
& +\frac{q^{2}}{8} B_{2}^{-1} \sin \left(2 \psi_{s}+2 \psi_{0}\right) .
\end{aligned}
$$

Finally

$$
E_{2}=\left[1-e^{\prime} \cos \left(M_{s}+E_{0}+E_{1}\right)\right]^{-1} M_{2}
$$

where

$$
\begin{aligned}
M_{2}= & -\left(a+b_{1}\right)^{-1}\left[A_{1} v_{1}+A_{11} \sin \left(M_{s}+v_{0}\right)+A_{12} \sin \left(2 M_{s}+2 v_{0}\right)\right. \\
& \left.+c^{2}\left(-2 \alpha_{1}\right)^{1 / 2}\left(\alpha_{2}^{2}-\alpha_{3}^{2}\right)^{-1 / 2} \eta_{0}^{3}\left\{B_{1} \psi_{1}-\frac{1}{2} \psi_{1} \cos \left(2 \psi_{s}+2 \psi_{0}\right)-\frac{q^{2}}{8} \sin \left(2 \psi_{s}+2 \psi_{0}\right)+\frac{q^{2}}{64} \sin \left(4 \psi_{s}+4 \psi_{0}\right)\right\}\right] .
\end{aligned}
$$

Then $v_{2}$ is found by placing $v=M_{s}+v_{0}+v_{1}+v_{2}$ and $E=M_{s}+E_{0}+E_{1}+E_{2}$ in the anomaly connections and

$$
\begin{aligned}
\psi_{2}=\left(-2 \alpha_{1}\right)^{-1 / 2}\left(\alpha_{2}^{2}-\alpha_{3}^{2}\right)^{1 / 2} \eta_{0}^{-1} B_{2}^{-1}\left[A_{2} v_{2}+A_{21} v_{1} \cos \left(M_{s}+v_{0}\right)\right. & +2 A_{22} v_{1} \cos \left(2 M_{s}+2 v_{0}\right) \\
\left.+A_{23} \sin \left(3 M_{s}+3 v_{0}\right)+A_{24} \sin \left(4 M_{s}+4 v_{0}\right)\right] & +\frac{q^{2}}{4} B_{2}^{-1}\left[\psi_{1} \cos \left(2 \psi_{s}+2 \psi_{0}\right)\right. \\
& \left.+\frac{3 q^{2}}{8} \sin \left(2 \psi_{s}+2 \psi_{0}\right)-\frac{3 q^{2}}{64} \sin \left(4 \psi_{s}+4 \psi_{0}\right)\right]
\end{aligned}
$$

\section{Unperturbed Reference Orbit}

The spheroidal coordinates $\rho$ and $\eta$ are then given by

$$
\rho=a(1-e \cos E)=(1+e \cos v)^{-1} p \quad \eta=\eta_{0} \sin \psi,
$$

where $E=M_{s}+E_{0}+E_{1}+E_{2}$ and $v=M_{s}+v_{0}+v_{1}+v_{2}$. To obtain the unperturbed right ascension $\phi$, first calculate an angle $\chi$, equaling $\psi$ whenever $\psi$ is a multiple of $\pi / 2$ and satisfying

$$
\cos \chi=\left(1-\eta_{0}^{2} \sin ^{2} \psi\right)^{-1 / 2} \cos \psi \quad \sin \chi=\left(1-\eta_{0}^{2}\right)^{1 / 2}\left(1-\eta_{0}^{2} \sin ^{2} \psi\right)^{-1 / 2} \sin \psi
$$

Then

$$
\begin{array}{r}
\phi=\beta_{3}+\alpha_{3}\left(\alpha_{2}^{2}-\alpha_{3}^{2}\right)^{-1 / 2} \eta_{0}\left[\left(1-\eta_{0}^{2}\right)^{-1 / 2}\left(1-\eta_{2}^{-2}\right)^{-1 / 2} \chi+B_{3} \psi+\frac{3}{32} \eta_{0}^{2} \eta_{2}^{-4} \sin 2 \psi\right] \\
-c^{2} \alpha_{3}\left(-2 \alpha_{1}\right)^{-1 / 2}\left[A_{3} v+\sum_{n=1}^{4} A_{3 n} \sin n v\right] .
\end{array}
$$

\section{The Zonal Harmonic Perturbations}

Within the accuracy of calculation of the perturbations, we may use either the approximate or the accurate formulas listed below. (Use of the approximate formulas may involve more work than 
use of the accurate ones, because the latter will already be known from the solution of the unperturbed problem.) Compute

\begin{tabular}{c|c|c}
\hline \hline & Approximate formula & Accurate formula \\
\hline$n$ & $\mu^{1 / 2} a^{-3 / 2}$ & \\
$l$ & $l_{0}+n t$ & $M_{s}=l_{0}+2 \pi \nu_{1} t$ \\
$g$ & $g_{0}+\frac{3}{4}\left(\frac{r_{e}}{p}\right)^{2} n J_{2}\left(5 \cos ^{2} I-1\right) t$ & $g_{0}+2 \pi\left(\nu_{2}-\nu_{1}\right) t$ \\
& $M_{s}+E_{0}$ & $M_{s}+E_{0}+E_{1}+E_{2}$ \\
$E$ & $M_{s}+v_{0}$ & $M_{s}+v_{0}+v_{1}+v_{2}$ \\
\hline
\end{tabular}

Also, $r=\rho$, within the accuracy of the calculation. Then compute

\section{Third Harmonic Short-Periodic Quantities}

$$
\begin{aligned}
L_{3}=-\frac{n r_{e}^{3}}{a}\left[\left(-\frac{3}{2} \eta_{0}+\frac{15}{8} \eta_{0}^{3}\right)\left\{\left(\frac{a}{r}\right)^{4} \sin (v+g)-e\left(1-e^{2}\right)^{-5 / 2} \sin g\right\}-\frac{5}{8} \eta_{0}^{3}\left(\frac{a}{r}\right)^{4} \sin (3 v+3 g)\right] \\
\begin{aligned}
G_{3}=-n a^{2}\left(1-e^{2}\right)^{1 / 2}\left(\frac{r_{e}}{p}\right)^{3}\left\{( - \frac { 3 } { 2 } \eta _ { 0 } + \frac { 1 5 } { 8 } \eta _ { 0 } ^ { 3 } ) \left[e(v-l) \cos g+\frac{e^{2}}{4} \sin (v-g)\right.\right. \\
\left.+\left(1+\frac{1}{2} e^{2}\right) \sin (v+g)+\frac{1}{2} e \sin (2 v+g)+\frac{e^{2}}{12} \sin (3 v+g)\right] \\
-\frac{15}{8} \eta_{0}^{3}\left[\frac{e^{2}}{4} \sin (v+3 g)+\frac{1}{2} e \sin (2 v+3 g)+\frac{1}{3}\left(1+\frac{1}{2} e^{2}\right) \sin (3 v+3 g)\right. \\
\left.\left.+\frac{e}{4} \sin (4 v+3 g)+\frac{e^{2}}{20} \sin (5 v+3 g)\right]\right\} .
\end{aligned}
\end{aligned}
$$

(Note that $v-l=v_{0}$, within the accuracy of the calculation.)

$$
\begin{aligned}
& l_{3}= \frac{3}{8 e}\left(1-e^{2}\right)^{1 / 2}\left(\frac{r_{e}}{p}\right)^{3} \eta_{0}\left(5 \eta_{0}^{2}-4\right)\left[\left(1-e^{2}\right)(v-l) \sin g+\left(1+\frac{1}{2} e^{2}\right) \cos g+\frac{e}{8}\left(14-3 e^{2}\right) \cos (v-g)\right. \\
&+ \frac{1}{2} e^{2} \cos (2 v-g)+\frac{e^{3}}{16} \cos (3 v-g)-e\left(1-e^{2}\right) \cos (v+g)-\frac{3}{2} \cos (2 v+g)-\frac{e}{24}\left(34-e^{2}\right) \cos (3 v+g) \\
&\left.-\frac{1}{2} e^{2} \cos (4 v+g)-\frac{e^{3}}{16} \cos (5 v+g)\right]+\frac{5}{8 e}\left(1-e^{2}\right)^{1 / 2}\left(\frac{r_{e}}{p}\right)^{3} \eta_{0}^{3}\left[-\frac{1}{2} e^{2} \cos 3 g-\frac{e^{3}}{16} \cos (v-3 g)\right. \\
&-+\frac{e}{8}\left(6+5 e^{2}\right) \cos (v+3 g)-\left(\frac{1}{2}+e^{2}\right) \cos (2 v+3 g)+\frac{e}{3}\left(1-e^{2}\right) \cos (3 v+3 g)+\frac{1}{4}\left(5+e^{2}\right) \cos (4 v+3 g) \\
& g_{3}=-\left(1-e^{2}\right)^{-1 / 2} l_{3}-\frac{3}{8}\left(\frac{r_{e}}{p}\right)^{3} \eta_{0}^{-1}\left(4-39 \eta_{0}^{2}+40 \eta_{0}^{4}\right)\left[e(v-l) \cos (5 v+3 g)+\frac{1}{2} e^{2} \cos (6 v+3 g)+\frac{e^{3}}{16} \cos (7 v+3 g)\right] \\
&\left.\quad-\left(1+\frac{1}{2} e^{2}\right) \cos (v+g)-\frac{1}{2} e \cos (2 v+g)-\frac{e^{2}}{12} \cos (3 v+g)\right] \\
& \quad-\frac{5}{8}\left(\frac{r_{e}}{p}\right)^{3} \eta_{0}\left(8 \eta_{0}^{2}-3\right)\left[\frac{e^{2}}{4} \cos (v+3 g)+\frac{1}{2} e \cos (2 v+3 g)+\frac{1}{3}\left(1+\frac{1}{2} e^{2}\right) \cos (3 v+3 g)\right. \\
&+\frac{e}{4} \cos (4 v+3 g)+\frac{e^{2}}{20} \cos (5 v+3 g)
\end{aligned}
$$




$$
\begin{aligned}
& h_{3}=\left(\frac{r_{e}}{p}\right)^{3}\left\{\frac{3}{2} \cot I\left(1-\frac{15}{4} \eta_{0}^{2}\right)[e(v-l)\right. \sin g+\frac{e^{2}}{4} \cos (v-g)-\left(1+\frac{1}{2} e^{2}\right) \cos (v+g) \\
&\left.-\frac{1}{2} e \cos (2 v+g)-\frac{e^{2}}{12} \cos (3 v+g)\right]-\frac{15}{16} \sin 2 I\left[\frac{e^{2}}{4} \cos (v+3 g)+\frac{1}{2} e \cos (2 v+3 g)\right. \\
&\left.\left.+\frac{1}{3}\left(1+\frac{1}{2} e^{2}\right) \cos (3 v+3 g)+\frac{e}{4} \cos (4 v+3 g)+\frac{e^{2}}{20} \cos (5 v+3 g)\right]\right\}
\end{aligned}
$$

\section{Third Harmonic Long-Periodic Quantities}

$$
\begin{aligned}
\tilde{G}_{3} & =\frac{1}{2} \dot{r}_{e} n a\left(1-e^{2}\right)^{-1 / 2} e \eta_{0} \sin g \\
\tilde{l}_{3} & =\frac{r_{e}}{2 a e}\left(1-e^{2}\right)^{1 / 2} \eta_{0} \cos g \\
\tilde{g}_{3} & =\frac{r_{e}}{2 p}\left(\frac{e \cos ^{2} I}{\sin I}-\frac{\sin I}{e}\right) \cos g \\
\tilde{h}_{3} & =-\frac{r_{e}}{2 p} e \cot I \cos g
\end{aligned}
$$

\section{Fourth Harmonic Short-Periodic Quantities}

$$
\begin{aligned}
& L_{4}=-\frac{1}{8}\left(\frac{r_{e}}{a}\right)^{4}(\mu a)^{1 / 2}\left\{\left(3-15 \eta_{0}^{2}+\frac{105}{8} \eta_{0}^{4}\right)\left[\left(\frac{a}{r}\right)^{5}-\left(1-e^{2}\right)^{-7 / 2}\left(1+\frac{3}{2} e^{2}\right)\right]\right. \\
& +5 \eta_{0}^{2}\left(3-\frac{7}{2} \eta_{0}^{2}\right)\left[\left(\frac{a}{r}\right)^{5} \cos (2 v+2 g)-\frac{3}{4} e^{2}\left(1-e^{2}\right)^{-7 / 2} \cos 2 g\right] \\
& \left.+\frac{35}{8} \eta_{0}^{4}\left(\frac{a}{r}\right)^{5} \cos (4 v+4 g)\right\} \\
& G_{4}=-\frac{5}{8}\left(\frac{r_{e}}{p}\right)^{4}(\mu p)^{1 / 2} \eta_{0}^{2}\left\{( 3 - \frac { 7 } { 2 } \eta _ { 0 } ^ { 2 } ) \left[-\frac{3}{2} e^{2}(v-l) \sin 2 g-\frac{e^{3}}{4} \cos (v-2 g)\right.\right. \\
& +\left(3 e+\frac{3 e^{3}}{4}\right) \cos (v+2 g)+\left(1+\frac{3}{2} e^{2}\right) \cos (2 v+2 g)+\left(e+\frac{e^{3}}{4}\right) \cos (3 v+2 g) \\
& \left.+\frac{3 e^{2}}{8} \cos (4 v+2 g)+\frac{e^{3}}{20} \cos (5 v+2 g)\right]+\frac{7}{8} \eta_{0}^{2}\left[\frac{1}{2} e^{3} \cos (v+4 g)+\frac{3 e^{2}}{2} \cos (2 v+4 g)\right. \\
& +\left(2 e+\frac{1}{2} e^{3}\right) \cos (3 v+4 g)+\left(1+\frac{3}{2} e^{2}\right) \cos (4 v+4 g)+\left(\frac{6 e}{5}+\frac{3 e^{3}}{10}\right) \cos (5 v+4 g) \\
& \left.\left.+\frac{1}{2} e^{2} \cos (6 v+4 g)+\frac{e^{3}}{14} \cos (7 v+4 g)\right]\right\} \\
& l_{41}=\left(\frac{r_{e}}{p}\right)^{4} \frac{\left(1-e^{2}\right)^{1 / 2}}{2048}\left\{6 ( 8 - 4 0 \eta _ { 0 } ^ { 2 } + 3 5 \eta _ { 0 } ^ { 4 } ) \left[48\left(1-e^{2}\right)(v-l)\right.\right.
\end{aligned}
$$$$
+\frac{2}{e}\left(40+12 e^{2}-17 e^{4}\right) \sin v+4\left(20-e^{2}\right) \sin 2 v+e\left(40-e^{2}\right) \sin 3 v+10 e^{2} \sin 4 v
$$$$
\left.+e^{3} \sin 5 v\right]-4 \eta_{0}^{2}\left(7 \eta_{0}^{2}-6\right)\left[240\left(1-e^{2}\right)(v-l) \cos 2 g+5 e^{3} \sin (3 v-2 g)\right.
$$$$
+50 e^{2} \sin (2 v-2 g)+15 e\left(16-3 e^{2}\right) \sin (v-2 g)-20\left(14+5 e^{2}\right) \sin 2 g
$$$$
+\frac{10}{e}\left(8-24 e^{2}-19 e^{4}\right) \sin (v+2 g)+240\left(1-e^{2}\right) \sin (2 v+2 g)+\frac{10}{e}\left(24+16 e^{2}-5 e^{4}\right) \sin (3 v+2 g)
$$$$
\left.+20\left(17+2 e^{2}\right) \sin (4 v+2 g)+3 e\left(64+e^{2}\right) \sin (5 v+2 g)+50 e^{2} \sin (6 v+2 g)+5 e^{3} \sin (7 v+2 g)\right]
$$ 


$$
\begin{aligned}
+ & \eta_{0}^{4}\left[35 e^{3} \sin (v-4 g)-350 e^{2} \sin 4 g-105 e\left(8+5 e^{2}\right) \sin (v+4 g)-140\left(8+11 e^{2}\right) \sin (2 v+4 g)\right. \\
- & \frac{70}{e}\left(8+20 e^{2}+7 e^{4}\right) \sin (3 v+4 g)+840\left(1-e^{2}\right) \sin (4 v+4 g)+\frac{14}{e}\left(104+84 e^{2}-13 e^{4}\right) \sin (5 v+4 g) \\
+ & 140\left(16+3 e^{2}\right) \sin (6 v+4 g)+15 e\left(88+3 e^{2}\right) \sin (7 v+4 g)+350 e^{2} \sin (8 v+4 g) \\
g_{41}= & \left.-\left(1-e^{2}\right)^{-1 / 2} l_{41}-\frac{\left(r_{e} / p\right)^{4}}{512}\left\{2\left(136-500 \eta_{0}^{2}+385 \eta_{0}^{4}\right)\left[6\left(2+3 e^{2}\right)(v-l)+4 g\right)\right]\right\} \\
+ & \left.9 e\left(4+e^{2}\right) \sin v+9 e^{2} \sin 2 v+e^{3} \sin 3 v\right]-2\left(12-82 \eta_{0}^{2}+77 \eta_{0}^{4}\right)\left[60 e^{2}(v-l) \cos 2 g\right. \\
+ & 10 e^{3} \sin (v-2 g)+30 e\left(4+e^{2}\right) \sin (v+2 g)+20\left(2+3 e^{2}\right) \sin (2 v+2 g)+10 e\left(4+e^{2}\right) \sin (3 v+2 g) \\
+ & \left.15 e^{2} \sin (4 v+2 g)+2 e^{3} \sin (5 v+2 g)\right]+\eta \eta^{2}\left(11 \eta^{2}-4\right)\left[35 e^{3} \sin (v+4 g)+105 e^{2} \sin (2 v+4 g)\right. \\
+ & 35 e\left(4+e^{2}\right) \sin (3 v+4 g)+35\left(2+3 e^{2}\right) \sin (4 v+4 g)+21 e\left(4+3 e^{2}\right) \sin (5 v+4 g) \\
& +7 \eta_{0}^{2}\left[\frac{e^{3}}{8} \sin (v+4 g)+\frac{3 e^{2}}{8} \sin (2 v+4 g)+\left(\frac{1}{2} e+\frac{e^{3}}{8}\right) \sin (3 v+4 g)+\left(\frac{1}{4}+\frac{3 e^{2}}{8}\right) \sin (4 v+4 g)\right. \\
& +\frac{5}{16}\left(\frac{r_{e}}{p}\right)^{4} \cos I\left\{3 ( 7 \eta _ { 0 } ^ { 2 } - 4 ) \left[\left(1+\frac{3}{2} e^{2}\right)(v-l)+\left(3 e+\frac{3}{4} e^{3}\right) \sin v+\frac{3}{4} e^{2} \sin 2 v\right.\right. \\
& \left.\left.+\frac{e^{3}}{12} \sin 3 v\right]+4\left(3-7 \eta_{0}^{2}\right)\left[\frac{3}{4} e^{2}(v-l) \sin (5 v+4 g)+\frac{e^{2}}{8} \sin (6 v+4 g)+\frac{e^{3}}{56} \sin (7 v+4 g)\right]\right\} \\
& \left.+\left(\frac{1}{2}+\frac{3 e^{2}}{4}\right) \sin (2 v+2 g)+\left(\frac{1}{2} e+\frac{e^{3}}{8}\right) \sin (3 v+2 g)+\frac{3 e^{2}}{16} \sin (4 v+2 g)+\frac{e^{3}}{40} \sin (5 v+2 g)\right] \\
h_{41}(v-2 g)+\left(\frac{3 e}{2}+\frac{3 e^{3}}{8}\right) \sin (v+2 g) & \\
& \left.\left.+5 e^{3} \sin (7 v+4 g)\right]\right\}
\end{aligned}
$$

Fourth Harmonic Long-Periodic Quantities

$$
\begin{aligned}
\tilde{G}_{4}= & -\frac{5 r_{e}^{2} n}{16} e^{2}\left(1-e^{2}\right)^{-3 / 2} \eta_{0}^{2}\left(1-7 \cos ^{2} I\right)\left(1-5 \cos ^{2} I\right)^{-1} \cos 2 g \\
\tilde{l}_{4}= & \frac{5}{16}\left(\frac{r_{e}}{a}\right)^{2}\left(1-e^{2}\right)^{-1 / 2} \eta_{0}^{2}\left(1-7 \cos ^{2} I\right)\left(1-5 \cos ^{2} I\right)^{-1} \sin 2 g \\
\tilde{g}_{4}= & -\frac{5}{32}\left(\frac{r_{e}}{p}\right)^{2}\left[2+e^{2}-3\left(2+3 e^{2}\right) \cos ^{2} I-8\left(2+5 e^{2}\right) \cos ^{4} I\left(1-5 \cos ^{2} I\right)^{-1}\right. \\
& \left.-80 e^{2} \cos ^{6} I\left(1-5 \cos ^{2} I\right)^{-2}\right] \sin 2 g \\
\tilde{h}_{4}= & -\frac{5}{16}\left(\frac{r_{e}}{p}\right)^{2} e^{2} \cos I\left[3+16 \cos ^{2} I\left(1-5 \cos ^{2} I\right)^{-1}+40 \cos ^{4} I\left(1-5 \cos ^{2} I\right)^{-2}\right] \sin 2 g .
\end{aligned}
$$

Fourth Harmonic Secular Quantities

$$
\begin{aligned}
& l_{42}=-\frac{45}{128}\left(\frac{r_{e}}{p}\right)^{4} n\left(1-e^{2}\right)^{1 / 2}\left(3-30 \cos ^{2} I+35 \cos ^{4} I\right) t \\
& g_{42}=-\frac{15}{128}\left(\frac{r_{e}}{p}\right)^{4} n\left[3\left(4+3 e^{2}\right)-18\left(8+7 e^{2}\right) \cos ^{2} I+7\left(28+27 e^{2}\right) \cos ^{4} I\right] t \\
& h_{42}=-\frac{15}{32}\left(\frac{r_{e}}{p}\right)^{4} n\left(2+3 e^{2}\right) \cos I\left(3-7 \cos ^{2} I\right) t .
\end{aligned}
$$


With $\sigma_{4} \equiv J_{4}+J_{2}^{2}$, next compute the variations in the Delaunay variables

$$
\begin{aligned}
& \delta L=J_{3} L_{3}+\sigma_{4} L_{4} \quad \delta H=0 \\
& \delta G=J_{3} G_{3}+\frac{J_{3}}{J_{2}} \tilde{G}_{3}+\sigma_{4} G_{4}+\frac{\sigma_{4}}{J_{2}} \tilde{G}_{4} \\
& \delta l=J_{3} l_{3}+\frac{J_{3}}{J_{2}} \tilde{l}_{3}+\sigma_{4}\left(l_{41}+l_{42}\right)+\frac{\sigma_{4}}{J_{2}} \tilde{l}_{4} \\
& \delta g=J_{3} g_{3}+\frac{J_{3}}{J_{2}} \tilde{g}_{3}+\sigma_{4}\left(g_{41}+g_{42}\right)+\frac{\sigma_{4}}{J_{2}} \tilde{g}_{4} \\
& \delta h=J_{3} h_{3}+\frac{J_{3}}{J_{2}} \tilde{h}_{3}+\sigma_{4}\left(h_{41}+h_{42}\right)+\frac{\sigma_{4}}{J_{2}} \tilde{h}_{4}
\end{aligned}
$$

The variations of the elements $a, e$, and $\eta_{0}$ are then

$$
\delta a=\frac{2}{a n} \delta L \quad \delta e=\frac{p n}{\mu e} \delta L-(a e)^{-1}\left(\frac{p}{\mu}\right)^{1 / 2} \delta G \quad \delta \eta_{0}=\frac{1-\eta_{0}^{2}}{\mu \eta_{0} p} \delta G
$$

The variations in the uniformising variables $E, v$, and $\psi$ are

$$
\begin{aligned}
& \delta E=(\rho / a)(\delta l+\sin E \delta e) \\
& \delta v=\left(1-e^{2}\right)^{1 / 2}(\rho / a)\left[\delta E+\left(1-e^{2}\right)^{-1} \sin E \delta e\right] \\
& \delta \psi=\delta v+\delta g
\end{aligned}
$$

The variations in the spheroidal coordinates $\rho, \eta$, and $\phi$ are then

$$
\begin{aligned}
& \delta \rho=(\rho / a) \delta a-a \cos E \delta e+a e \sin E \delta E \\
& \delta \eta=\left(\eta / \eta_{0}\right) \delta \eta_{0}+\eta_{0} \cos \psi \delta \psi \\
& \delta \phi=\delta h+\left(1-\eta_{0}^{2} \sin ^{2} \psi\right)^{-1} \cos I\left[\delta \psi-\frac{1}{2}(\mu p)^{-1 / 2} \sin 2 \psi \delta G\right]
\end{aligned}
$$

The final rectangular coordinates $X+\delta X, Y+\delta Y$, and $Z+\delta Z$ are then given by

$$
\begin{gathered}
X+\delta X+i(Y+\delta Y)=\left[(\rho+\delta \rho)^{2}+c^{2}\right]^{1 / 2}\left[1-(\eta+\delta \eta)^{2}\right]^{1 / 2} \exp i(\phi+\delta \phi) \\
\delta Z=\rho \delta \eta+\eta \delta \rho .
\end{gathered}
$$

\section{Discussion of Results}

Since the chosen accuracy of the quantities appearing in the present perturbation, with factor of order $J_{2}^{2}$, is only that of an elliptic approximation, the variations in the Delaunay variables may be checked against those found by Brouwer [1959], by Garfinkel [1959], or by Kozai [1962]. Comparison of the above results with those of Brouwer or of Garfinkel shows that the long-periodic effects of the third and fourth harmonics and the secular effects of the fourth agree with Brouwer's or Garfinkel's, provided that one replaces their $J_{4}$ by $J_{4}+J_{2}^{2}$. Comparison with Kozai shows that the short-periodic effects agree with his. ${ }^{1}$ Similarly one can read out of Kozai's paper the longperiodic effects of $J_{5}, J_{7}$, and $J_{9}$ and the long-periodic and secular effects of $J_{6}$ and $J_{8}$; in so doing one ought in principle to replace his $J_{6}$ by $J_{6}-J_{2}^{3}$ and his $J_{8}$ by $J_{8}+J_{2}^{4}$, but this would be going beyond the accuracy of the present calculation. Since the author's orbital elements differ from Kozai's by terms of order $J_{2}$, the agreement with Kozai holds only through terms of order $J_{2}$ for long-periodic effects and through terms of order $J_{2}^{2}$ for short-periodic effects.

${ }^{1}$ On his page 451 , however, in the first line for $\Delta G$, the expression $\cos 2 g$ should be $\cos g$. 
To compare accuracies, we construct the following table, noting that the author's reference orbit accounts for about 99.5 percent of the deviation of the earth's potential from spherical symmetry. Thus my perturbation potential is only about 0.5 percent of Kozai's.

\begin{tabular}{c|c|c|c}
\hline \hline \multicolumn{4}{c}{ Effects of 99.5 percent of deviation from sphericity } \\
\hline \multirow{3}{*}{$\begin{array}{c}\text { Kozai } \\
\text { Author }\end{array}$} & $\begin{array}{c}\text { Secular accuracy } \\
\text { Through } J_{2}^{3} \\
\text { Exact }\end{array}$ & $\begin{array}{c}\text { Short-periodic accuracy } \\
\text { Through } J_{2}^{2}\end{array}$ & $\begin{array}{c}\text { Long-periodic accuracy } \\
\text { Terms do not exist } \\
\text { Through } J_{2}^{2}\end{array}$ \\
\hline
\end{tabular}

Effects of remaining 0.5 percent of deviation from spherity

\begin{tabular}{c|c|c|c}
\hline & Secular accuracy & Short-periodic accuracy & Long-periodic accuracy \\
Kozai & Through $J_{2}^{3}$ & Through $J_{2}^{2}$ & Through $J_{2}^{2}$ \\
Author & Through $J_{2}^{2}$ & Through $J_{2}^{2}$ & Through $J_{2}^{2}$ \\
\hline
\end{tabular}

Thus the advantages of the author's treatment are the exact solution for the secular effects arising from 99.5 percent of the aspherical deviation and the much shorter algorithm. The principal advantage of Kozai's treatment, arising in connection with the remaining 0.5 percent of the aspherical deviation, is his more accurate solution for the long-periodic terms.

The present solution, like all previous perturbation theories, gives rise to the resonance denominator 1-5 $\cos ^{2} I$ in some of the long-periodic terms. These terms are thus not reliable if one considers inclinations $I$ sufficiently close to $63.4^{\circ}$ or $116.6^{\circ}$. For such inclinations one could improve the accuracy by boldly dropping the long-periodic terms with coefficient $J_{4}+J_{2}^{2}$ or, better, by superposing on the present treatment Izsak's [1962] solution of the problem of the critical inclination.

The element $e$ occurs in the denominators of $\delta e, l_{3}, g_{3}, \tilde{l}_{3}, \tilde{g}_{3}, l_{41}$, and $g_{41}$ and thus also in the denominators of $\delta l, \delta g, \delta E, \delta v$, and $\delta \psi$. No corresponding trouble occurs in the coordinates, however. To test this point, reject all terms except those containing $e^{-1}$. One then finds $l_{3}=$ $-g_{3}, \tilde{l}_{3}=-\tilde{g}_{3}, l_{41}=-g_{41}, L_{3}=G_{3}, L_{4}=G_{4}$, and $\tilde{G}_{3}=\tilde{G}_{4}=0$. Then $\delta e=O\left(e^{0}\right), \delta E=\delta v=\delta l$, and $\delta \psi=\delta v+\delta g=\delta l+\delta g=0$. Trouble could occur in $\delta \eta$ or in $\delta \phi$ only through $\delta \psi$, so that $\delta \eta$ and $\delta \phi$ do not become infinite for $e=0$. Similarly, trouble could occur in $\delta \rho$ through the term $-a \cos E \delta e$, which, however, does not become infinite, or through the term $a e \sin E \delta E$. But $\delta E=\delta l$ $=O\left(e^{-1}\right)$, so that this term also remains finite.

The quantity $\eta_{0} \equiv \sin I$ occurs in the denominators of $\delta \eta_{0}, g_{3}, h_{3}, \tilde{g}_{3}$, and $\tilde{h}_{3}$, and thus also in the denominators of $\delta g, \delta h, \delta \psi$, and $\delta \phi$. Again, however, the coordinates remain finite when $\sin I=0$. To test this point, reject all terms except those containing $\eta_{0}^{-1}=\csc I$. Since $G_{3}$ and $\tilde{G}_{3}$ are of order $\eta_{0}$ and $G_{4}$ and $\tilde{G}_{4}$ of order $\eta_{0}^{2}$, it follows that $\delta G$ is also of order $\eta_{0}$ and $\delta \eta_{0}=\left(1-\eta_{0}^{2}\right) \delta G /\left(\eta_{0} \mu p\right)$ remains finite. Thus the term $\sin \psi \delta \eta_{0}$ in $\delta \eta$ remains finite. We also have $g_{3}=-h_{3}=O\left(\eta_{0}^{-1}\right), \tilde{g}_{3}=\frac{1}{2} e r_{e} p^{-1}$ $\times \cos ^{2} I \csc I \cos g, \tilde{h}_{3}=-\frac{1}{2} e r_{e} p^{-1} \cos I \csc I \cos g$, and $\delta \psi=\delta g=O\left(\eta_{0}^{-1}\right)$. Thus the term $\eta_{0} \cos \psi \delta \psi$ in $\delta \eta$ also remains finite, so that $\delta \eta$ remains finite. Finally, $\delta \phi=\delta h+\cos I \delta g=J_{3}\left(g_{3}+h_{3}\right)+J_{3} J_{2}^{-1}\left(\tilde{h}_{3}\right.$ $\left.+\tilde{g}_{3} \cos I\right)=\frac{1}{2} J_{3} J_{2}^{-1} e r_{e} p^{-1} \cos g \csc I\left(\cos ^{3} I-\cos I\right)=-\frac{1}{2} J_{3} J_{2}^{-1} e r_{e} p^{-1} \sin I \cos I \cos g=0$. Thus $\delta \phi$ remains finite. There is no trouble with $\delta \rho$. 


\section{References}

Brouwer, D. 1959, Astron J. 64, 378-397.

Garfinkel, B. 1959, Astron. J. 64, 353-367.

Garfinkel, B. 1960, Notes of the Summer Institute in Dynamical Astronomy at Yale University, July 1960, page 281.

Izsak, I. 1962, Smithsonian Astrophysical Observatory, Special Report No. 60.

Kaula, W. 1962, Advances in Geophysics, Vol. IX, edited by H. E. Landsberg and J. Van Mieghem, p. 225 (Academic Press).

King-Hele, D. G., C. E. Cook, and J. M. Rees, 1963, Nature 197, 785.

Langer, R. M. and J. P. Vinti, (1963) J. Res. NBS 67C (Eng. and Instr.) No. 3, 247-249.

Vinti, J. P. 1959a, Phys. Rev. Letters 3, 8; 1959b, J. Res. NBS 63B (Math. and Math. Phys.) No. 2, 105-116; 1961a, J. Res. NBS 65B (Math. and Math. Phys.) No. 3, 169-201; 1961b, Astron. J. 66, 514-516; 1962, J. Res. NBS, 66B (Math. and Math. Phys.) No. 1, 5-13.

(Paper 67B4-103) 